\title{
General Method of Construction of Implicit Discrete Maps Generating Chaos in 3D Quadratic Systems of Differential Equations
}

\author{
Vasiliy Ye. Belozyorov \\ Department of Applied Mathematics, \\ Dnepropetrovsk National University, \\ Gagarin's Avenue, 72, \\ 49050, Dnepropetrovsk, Ukraine \\ belozvye@mail.ru
}

Received August 14, 2013; Revised October 13, 2013

\begin{abstract}
A method allowing to study the dynamics of 3D systems of quadratic differential equations by the reduction of these systems to the special $2 \mathrm{D}$ systems is presented. The mentioned $2 \mathrm{D}$ systems are used for the construction of new types of discrete maps generating the chaotic dynamics in some 3D autonomous systems of quadratic differential equations. Strong simplification of all results gives an introduction of the Lambert function. Due to this function some implicit discrete maps become explicit. Examples are given.
\end{abstract}

Keywords: Implicit 1D and 2D discrete maps; ordinary autonomous differential equations 3D system; limit cycle; chaotic attractor; Lambert function.

\section{Introduction}

The traditional research method of chaotic systems is that, for a given dynamical system, one can judge it is chaotic or not by using various methods such as the various definitions or characteristic quantities of chaos, this process is being passively discovered. However, since they can be widely applied, more chaos systems need be found or created actively to meet the needs of engineering applications (see, for example, [Belozyorov, 2011b; Belozyorov, 2012; Belozyorov \& Chernyshenko, 2013; Feng \& Tse, 2007; Khan \& Kumar, 2013; Kocan, 2012; Luo \& Guo, 2010; Robinson, 2004; Shen \& Jia, 2011; Zhang et al., 2009], and many references cited therein).

There are two basic methods to search for chaotic systems. They are either based on establishing a homoclinic orbit or by constructing the given system of a discrete map and its proof of being chaotic.
There are several papers devoted to the search of homoclinic orbits in 3D systems of differential equations (see, for example, [Belozyorov, 2011a; Belozyorov \& Chernyshenko, 2013; Chen et al., 2009; El-Dessoky et al., 2012; Jiang \& Sun, 2007; Bao \& Yang, 2011; Leonov \& Kuznetsov, 2013; Peng, 2008; Shang \& Han, 2005; Wang, 2009; Yang et al., 2010; Zheng \& Chen, 2006]). In our opinion, one of the most essential results was obtained in [Leonov, 2012; Leonov, 2013]. In these articles, the Fishing Principle allowing to decide a question about the existence of homoclinic orbits for a large class of nonlinear systems was offered. The construction of discrete maps for continuous dynamic systems is a less studied domain. Here the basic results are contained, for example, in [Belozyorov, 2011a; Belozyorov \& Chernyshenko, 2013; Gardini et al., 2011; Khan \& Kumar, 2013; Kocan, 2012; Luo \& Guo, 2010; Zhang et al., 2009]. 
We note that the existence (or absence) of chaos in a continuous dynamical system can be easily proved, if the Poincaré section of a phase flow of this system can be built successfully. Thus, we get $1 \mathrm{D}$ or $2 \mathrm{D}$ discrete map, the study of which strongly simplifies the research in the dynamics of the continuous system.

The most general approach in the study of chaos of the continuous 3D system consists in finding a basin of attraction for this system. The simplest situation arises then, when the basin of attraction is the entire space $\mathbb{R}^{3}$. In other words, for the existence of the basin it is sufficient that all solutions of the system are bounded for any initial data.

Currently, necessary and sufficient conditions of boundedness of all solutions for any quadratic 3D system of ordinary autonomous differential equations are unknown. At the same time, the authors of [Dickson \& Perko, 1970] assert that for 2D quadratic systems of ordinary autonomous differential equations, such conditions are found.

Below it will be shown that the boundedness of solution conditions for 2D systems derived in [Dickson \& Perko, 1970] are only sufficient. Nevertheless, these conditions appeared enough so that research of boundedness of solutions for 3D quadratic systems could be reduced to research of boundedness of solutions for $2 \mathrm{D}$ quadratic systems.

The present work is a continuation of the papers [Belozyorov, 2011a; Belozyorov \& Chernyshenko, 2013]. As compared to the paper [Belozyorov \& Chernyshenko, 2013] this article has substantial differences.

- In the considered systems, the existence of homoclinic orbits is not required.

- All considered discrete maps are exponential or polynomial (implicit or not).

- The boundedness of solution conditions of quadratic 3D systems of ordinary autonomous differential equations are more weak than in the article [Belozyorov, 2011a].

Consider 2D system of autonomous quadratic differential equations

$$
\left\{\begin{aligned}
\dot{x}(t)= & a_{11} x(t)+a_{12} y(t)+b_{11} x^{2}(t) \\
& +b_{12} x(t) y(t)+b_{22} y^{2}(t) \\
\dot{y}(t)= & a_{21} x(t)+a_{22} y(t)+c_{11} x^{2}(t) \\
& +c_{12} x(t) y(t)+c_{22} y^{2}(t)
\end{aligned}\right.
$$

where $a_{11}, \ldots, c_{22}$ are known real numbers. (At least one of the numbers $b_{11}, \ldots, b_{22}, c_{11}, \ldots, c_{22}$ is not equal to zero.) In the work [Dickson \& Perko, 1970] algebraic conditions of boundedness of solutions of system (1) were found. The essence of these conditions consists of the following.

Assume that there exist a linear nonsingular transformation $x \rightarrow s_{11} x+s_{12} y, y \rightarrow s_{21} x+s_{22} y$ $\left(s_{11} s_{22}-s_{21} s_{12} \neq 0\right)$ such that in new variables system (1) adopts one of the following three types:

$$
\left\{\begin{array}{l}
\dot{x}(t)=a_{11} x(t)+a_{12} y(t), \\
\dot{y}(t)=a_{21} x(t)+a_{22} y(t)+x(t) y(t),
\end{array}\right.
$$

where $a_{11}<0, a_{12}=0$ and $a_{22} \leq 0$;

$$
\left\{\begin{array}{l}
\dot{x}(t)=a_{11} x(t)+a_{12} y(t)+y^{2}(t), \\
\dot{y}(t)=a_{21} x(t)+a_{22} y(t),
\end{array}\right.
$$

where $a_{11} \leq 0, a_{21}=0, a_{22} \leq 0$ and $a_{11}+a_{22}<0$;

$$
\left\{\begin{aligned}
\dot{x}(t)= & a_{11} x(t)+a_{12} y(t)+y^{2}(t), \\
\dot{y}(t)= & a_{21} x(t)+a_{22} y(t)-x(t) y(t) \\
& +c_{22} y^{2}(t), \quad\left|c_{22}\right|<2,
\end{aligned}\right.
$$

where either (i) $a_{11}<0$; or (ii) $a_{11}=0$ and $a_{21}=0$; or (iii) $a_{11}=0, a_{21} \neq 0, a_{12}+a_{21}=0$ and $c_{22} a_{21}+a_{22} \leq 0$. (Notice that for simplification of designations in systems (2)-(4), we left those designations as in system (1).)

Theorem 1 [Dickson \& Perko, 1970, Theorem 1]. The quadratic system (1) has all of its trajectories bounded for $t \geq 0$ if and only if there exists a linear transformation which reduces it to one of systems (2), (3) or (4).

Consider the system

$$
\left\{\begin{aligned}
\dot{x}(t)= & a_{11} x(t)+a_{12} y(t)+b_{11} x^{2}(t) \\
& +b_{12} x(t) y(t)+b_{22} y^{2}(t), \\
\dot{y}(t)= & a_{21} x(t)+a_{22} y(t)+c_{12} x(t) y(t) \\
& +c_{22} y^{2}(t),
\end{aligned}\right.
$$

where $a_{21} \neq 0$.

In [Belozyorov, 2011a], Theorem 4 was proved for which system (5) appears as:

Theorem 2 [Belozyorov, 2011a, Theorem 4]. Assume that for system (5) we have:

(i) quadratic form $c_{12}\left(b_{11}-c_{12}\right) x^{2}+c_{12}\left(b_{12}-\right.$ $\left.c_{22}\right) x y+c_{12} b_{22} y^{2}$ is negative definite;

(ii) $b_{11}\left(b_{11}-c_{12}\right)<0$. 
Then for any initial values all solutions of system (5) are bounded.

It is easy to check that Theorems 1 and 2 are not implications of each other. Thus, the boundedness of solution conditions of quadratic $2 \mathrm{D}$ systems represented in Theorem 1 is not complete. In the future, Theorem 1 will be used for the proof of boundedness of solutions of quadratic 3D systems and the construction of $2 \mathrm{D}$ implicit discrete maps. For this purpose, we will retain one of the variables $x, y, z$ (for example $x$ ) fixed, and in the plane $y z$ formed by the coordinate axes $y$ and $z$, we will introduce the polar coordinates $\rho$ and $\phi$. A similar approach was used in [Gunay \& Kilic, 2011].

\section{Bounded Solutions of Quadratic 3D Systems of Differential Equations}

We will consider the following system:

$$
\left\{\begin{aligned}
\dot{x}(t)= & a_{11} x(t)+a_{12} y(t)+a_{13} z(t) \\
& +b_{11} y^{2}(t)+b_{12} y(t) z(t)+b_{22} z^{2}(t), \\
\dot{y}(t)= & a_{21} x(t)+a_{22} y(t)+a_{23} z(t) \\
& +c_{11} x(t) y(t)+c_{12} x(t) z(t), \\
\dot{z}(t)= & a_{31} x(t)+a_{32} y(t)+a_{33} z(t) \\
& +c_{21} x(t) y(t)+c_{22} x(t) z(t) .
\end{aligned}\right.
$$

(The research of such systems was started in [Belozyorov, 2011b].)

Introduce the restriction: the matrix

$$
A=\left(\begin{array}{ll}
a_{22} & a_{23} \\
a_{32} & a_{33}
\end{array}\right)
$$

has the negative discriminant Disc $=(\operatorname{tr} A)^{2}-4$ $\operatorname{det} A<0$.

Taking into account the last condition, system (6) by suitable linear real replacements of variables $y \rightarrow f_{11} y+f_{12} z$ and $z \rightarrow f_{21} y+f_{22} z$ can be transformed to the form

$$
\left\{\begin{aligned}
\dot{x}(t)= & a_{11} x(t)+a_{12} y(t)+a_{13} z(t) \\
& +b_{11} y^{2}(t)+b_{12} y(t) z(t)+b_{22} z^{2}(t), \\
\dot{y}(t)= & a_{21} x(t)+a_{22} y(t)+a_{23} z(t) \\
& +c_{11} x(t) y(t)+c_{12} x(t) z(t) \\
\dot{z}(t)= & a_{31} x(t)-a_{23} y(t)+a_{22} z(t) \\
& +c_{21} x(t) y(t)+c_{22} x(t) z(t) .
\end{aligned}\right.
$$

(For simplification of a further presentation, we retain for system (7) designations of system (6); it must not result in a misunderstanding, because system (6) is not used.)

Theorem 3. Suppose that for system (7) the following conditions are fulfilled:

(i) $a_{11}<0$;

(ii) the quadratic form $h(y, z)=b_{11} y^{2}+b_{12} y z+$ $b_{22} z^{2} \not \equiv 0$ is positive definite (negative definite);

(iii) the quadratic form $g(y, z)=c_{11} y^{2}+\left(c_{12}+\right.$ $\left.c_{21}\right) y z+c_{22} z^{2}$ is negative definite (positive definite).

Then for any initial values and $\forall t \geq 0$ the solutions $x(t), y(t), z(t)$ of system (7) are bounded.

Proof. Let us for system (7) define new variables $\rho \geq 0$ and $\phi$ by the formulas: $y=\rho \cos \phi, z=$ $\rho \cos \phi$. Then we obtain the new system

$$
\left\{\begin{aligned}
\dot{x}(t)= & a_{11} x+\left(a_{12} \cos \phi+a_{13} \sin \phi\right) \rho \\
& +\left(b_{11} \cos ^{2} \phi+b_{12} \cos \phi \sin \phi\right. \\
& \left.+b_{22} \sin ^{2} \phi\right) \rho^{2} \\
\dot{\rho}(t)= & \left(a_{21} \cos \phi+a_{31} \sin \phi\right) x+a_{22} \rho \\
& +\left[c_{11} \cos ^{2} \phi+\left(c_{12}+c_{21}\right) \cos \phi \sin \phi\right. \\
& \left.+c_{22} \sin ^{2} \phi\right] x \rho \\
\dot{\phi}(t)= & -a_{23}+\left[c_{21} \cos ^{2} \phi+\left(c_{22}-c_{11}\right)\right. \\
& \left.\times \cos \phi \sin \phi-c_{12} \sin ^{2} \phi\right] x \\
& +\left(a_{31} \cos \phi-a_{21} \sin \phi\right)\left(\frac{x}{\rho}\right) .
\end{aligned}\right.
$$

Without loss of generality, we can consider that the quadratic form $h(y, z)$ is positive definite and the quadratic form $g(y, z)$ is negative definite.

Let $x_{0} \geq 0$. Note that the case $x_{0} \leq 0$ has been taken previously. Really, let $x_{0} \leq 0$. If non-negative function $\exp \left(-a_{11} t\right) h(y(t), z(t))$ increases, then we see moment $T>0$ such that $\forall t>T$

$$
\begin{aligned}
x(t)= & \exp \left(a_{11} t\right)\left(x_{0}+\int_{T}^{t} \exp \left(-a_{11} \tau\right)\right. \\
& \times\left[\left(a_{12} \cos \phi(\tau)+a_{13} \sin \phi(\tau)\right) \rho(\tau)\right. \\
& +h(y(\tau), z(\tau))] d \tau) \geq 0 ;
\end{aligned}
$$


therefore, we have $x(t)>0$. If there is a moment $T_{1}>0$ such that $\forall t>T_{1}$ the function $\exp \left(-a_{11} t\right) h(y(t), z(t))$ decreases, then $\lim _{t \rightarrow \infty} x(t)=0$.

Let us find a minimum and a maximum of function $h=h(\phi) \equiv b_{11} \cos ^{2} \phi+b_{12} \cos \phi \sin \phi+b_{22} \sin ^{2} \phi$. Then we will have

$$
\begin{gathered}
h_{\min }=\frac{b_{11}+b_{22}-\sqrt{\left(b_{11}-b_{22}\right)^{2}+b_{12}^{2}}}{2} \geq 0, \\
h_{\max }=\frac{b_{11}+b_{22}+\sqrt{\left(b_{11}-b_{22}\right)^{2}+b_{12}^{2}}}{2}>0 .
\end{gathered}
$$

A similar research for the function $g=g(\phi) \equiv$ $c_{11} \cos ^{2} \phi+\left(c_{12}+c_{21}\right) \cos \phi \sin \phi+c_{22} \sin ^{2} \phi$ gives the following results:

$$
\begin{gathered}
g_{\max }=\frac{c_{11}+c_{22}+\sqrt{\left(c_{11}-c_{22}\right)^{2}+\left(c_{12}+c_{21}\right)^{2}}}{2}<0 \\
g_{\text {min }}=\frac{c_{11}+c_{22}-\sqrt{\left(c_{11}-c_{22}\right)^{2}+\left(c_{12}+c_{21}\right)^{2}}}{2}<0 .
\end{gathered}
$$

In addition, the following inequality is obvious:

$$
-\sqrt{p^{2}+q^{2}} \leq p \cos \phi+q \sin \phi \leq \sqrt{p^{2}+q^{2}} .
$$

Taking into account these remarks, we will have:

$$
\begin{aligned}
& a_{11} x+\left(a_{12} \cos \phi+a_{13} \sin \phi\right) \rho+\left(b_{11} \cos ^{2} \phi\right. \\
&\left.+b_{12} \cos \phi \sin \phi+b_{22} \sin ^{2} \phi\right) \rho^{2} \\
& \leq a_{11} x+\sqrt{a_{12}^{2}+a_{13}^{2}} \rho+h_{\max } \rho^{2}, \\
&\left(a_{21} \cos \phi+a_{31} \sin \phi\right) x+a_{22} \rho \\
&+\left[c_{11} \cos ^{2} \phi+\left(c_{12}+c_{21}\right) \cos \phi \sin \phi\right. \\
&\left.+c_{22} \sin ^{2} \phi\right] x \rho \\
& \leq \sqrt{a_{21}^{2}+a_{31}^{2}} x+a_{22} \rho+g_{\max } x \rho .
\end{aligned}
$$

We introduce the differentiated functions $x_{1}(t)$ and $\rho_{1}(t)$ satisfying the following system of differential equations:

$$
\left\{\begin{aligned}
\dot{x}_{1}(t)= & a_{11} x_{1}(t)+\sqrt{a_{12}^{2}+a_{13}^{2}} \rho_{1}(t) \\
& +h_{\max } \cdot \rho_{1}^{2}(t), \\
\dot{\rho}_{1}(t)= & \sqrt{a_{21}^{2}+a_{31}^{2}} x_{1}(t)+a_{22} \rho_{1}(t) \\
& +g_{\max } \cdot x_{1} \rho_{1}(t) .
\end{aligned}\right.
$$

According to Comparison Principle [Kuznetsov, 1998] for systems (8) and (9), we have: $x(t) \leq$ $x_{1}(t)$ and $\rho(t) \leq \rho_{1}(t)$. Thus, from boundedness of solutions of system (9) it follows that solutions $x(t)$ and $\rho(t)$ of system (8) are also bounded. (Generally speaking, angle $\phi$ can be unbounded.)

It is easy to check from Theorem 1 that condition $h_{\max } g_{\max }<0$, is necessary for boundedness of solutions of system (9). This condition has been proved in work [Belozyorov, 2011a]; the proof is based on the research of a homogeneous system (in (9) a linear part is absent). (In our case, the condition $h_{\max } g_{\max }<0$ is always satisfied.)

In [Belozyorov, 2011b] the condition of boundedness of solutions for a quadratic system of differential equations with almost any linear part is also met. For the systems of second order, this condition appeared very restrictive. Therefore, we will take advantage of Theorem 1, which asserts that for the boundedness of solutions of system (9), besides the condition $h_{\max } g_{\max }<0$, there is enough implementation of another condition: $a_{11}<0$. This completes the proof of Theorem 3 .

Further, we assume in system (8), $c_{11}=c_{22}=$ $p, c_{12}=-c_{21}=q, a_{11}=a, a_{22}=b, a_{23}=c$, $a_{12}=a_{13}=a_{21}=a_{31}=0$. Then, we get

$$
\left\{\begin{array}{l}
\dot{x}(t)=a x(t)+b_{11} y^{2}(t)+b_{12} y(t) z(t)+b_{22} z^{2}(t), \\
\dot{y}(t)=b y(t)+c z(t)+x(t)(p y(t)+q z(t)), \\
\dot{z}(t)=-c y(t)+b z(t)+x(t)(-q y(t)+p z(t)) .
\end{array}\right.
$$

This system will be used for establishing the chaotic properties of system (7).

\section{On Existence of Limit Cycles in System (10)}

Let

$$
\begin{gathered}
\dot{\mathbf{x}}(t)=\mathbf{G}(\mathbf{x}(t)), \quad \mathbf{x}(t) \in \mathbb{R}^{n}, \\
\mathbf{G}(\mathbf{x}) \in \mathbb{R}^{n}, \quad t \in \mathbb{R}
\end{gathered}
$$

be a system of ordinary autonomous differential equations and let $\mathbf{x}\left(t, \mathbf{x}_{0}\right)$ be a trajectory of this system with initial data $\mathbf{x}_{0} \in \mathbb{R}^{n}$. Here $\mathbf{G}(\mathbf{x}): \mathbb{R}^{n} \rightarrow$ $\mathbb{R}^{n}$ is a continuous vector-function; $\mathbf{x}\left(0, \mathbf{x}_{0}\right)=\mathbf{x}_{0}$.

The trajectory $\mathbf{x}\left(t, \mathbf{x}_{0}\right)$ of system (11) is called periodic if there exists a constant $T>0$ such that

$$
\forall t \in \mathbb{R} \quad \mathbf{x}\left(t+T, \mathbf{x}_{0}\right)=\mathbf{x}\left(t, \mathbf{x}_{0}\right) .
$$


Let $\mathbf{x}\left(t, \mathbf{y}_{0}\right) \quad\left(\mathbf{x}_{0} \neq \mathbf{y}_{0}\right)$ be another trajectory of system (11) such that $\left\|\mathbf{x}_{0}-\mathbf{y}_{0}\right\|<\epsilon$, where the symbol $\|\mathbf{v}\|$ means the Euclidean norm of the vector $\mathbf{v} ; \epsilon \rightarrow 0$ is any positive sufficiently small number.

The periodic trajectory $\mathbf{x}\left(t, \mathbf{x}_{0}\right)$ of system (11) is called isolated if for any positive sufficiently small number $\epsilon>0$ there does not exist the periodic trajectory $\mathbf{x}\left(t, \mathbf{y}_{0}\right)$ such that $\left\|\mathbf{x}_{0}-\mathbf{y}_{0}\right\|<\epsilon$. The isolated periodic trajectory $\mathbf{x}\left(t, \mathbf{x}_{0}\right)$ of system (11) is called a limit cycle.

A set $\mathbb{M} \subset \mathbb{R}^{n}$ is said to be a positively invariant set with respect to (11) if from $\mathbf{x}_{0} \in \mathbb{M}$ it follows that $\mathbf{x}\left(t, \mathbf{x}_{0}\right) \in \mathbb{M} \forall t \geq 0$.

A point $\mathbf{s} \in \mathbb{R}^{n}$ is said to be a positive limit point of $\mathbf{x}\left(t, \mathbf{x}_{0}\right)$ if there is a sequence $\left\{t_{m}\right\}$, with $t_{m} \rightarrow \infty$ as $m \rightarrow \infty$, such that $\mathbf{x}\left(t_{m}, \mathbf{x}_{0}\right) \rightarrow \mathbf{s}$ as $m \rightarrow \infty$. The set $\mathbb{L}^{+}$of all positive limit points of $\mathbf{x}\left(t, \mathbf{x}_{0}\right)$ is called the positive limit set of $\mathbf{x}\left(t, \mathbf{x}_{0}\right)$.

Let $\mathbb{D} \subset \mathbb{R}^{n}$ be a compact set.

Lemma 1 [Khalil, 1996, Lemma 3.1]. If solution $\mathbf{x}\left(t, \mathbf{x}_{0}\right)$ is bounded and belongs to $\mathbb{D}$, then its positive limit set $\mathbb{L}^{+}$is a nonempty, compact, invariant set. Moreover, $\mathbf{x}\left(t, \mathbf{x}_{0}\right) \rightarrow \mathbb{L}^{+}$as $t \rightarrow \infty$.

The following theorem is the substantial strengthening of Theorem 3 which was represented in [Belozyorov, 2011b].

Theorem 4. For system $(10)$ let $b>0,\left(b_{11}-b_{12}\right)^{2}+$ $b_{12}^{2} \neq 0$ and the conditions of Theorem 3 be valid. Then in system (10) there exists either a limit cycle or a limit torus.

Proof. Two different ways of the proof are possible: $q=0$ and $q \neq 0$. The case $q=0$ is indicated in the corollary of Theorem 5 . Therefore we will consider that $q \neq 0$.

Introduce into system (10) new variables $\rho$ and $\phi$ under the formulas: $y=\rho \cos \phi, z=\rho \sin \phi$, where $\rho>0$. Then, after replacement of variables and multiplication of the second and third equations of system (10) on the matrix

$$
\left(\begin{array}{cc}
\cos \phi(t) & \sin \phi(t) \\
-\frac{\sin \phi(t)}{\rho(t)} & \frac{\cos \phi(t)}{\rho(t)}
\end{array}\right),
$$

we get

$$
\left\{\begin{array}{l}
\dot{x}(t)=a \cdot x(t)+\rho^{2}(t) \cdot h(\phi(t)), \\
\dot{\rho}(t)=(b+p \cdot x(t)) \cdot \rho(t), \\
\dot{\phi}(t)=-(c+q \cdot x(t)) .
\end{array}\right.
$$

(A) From the second and third equations of system (12) it follows that

$$
\frac{d \rho}{\rho}+\frac{p}{q} d \phi=\left(b-c \frac{p}{q}\right) d t
$$

and

$$
\rho(\phi, t)=\rho_{0} \exp \left(-\frac{p}{q} \phi+\left(b-\frac{p}{q} c\right) t\right)
$$

where $\rho_{0}=\rho_{0}\left(\phi_{0}\right)=$ const $>0$.

In this case, system (12) may be reduced to the form

$$
\left\{\begin{aligned}
\dot{x}(t)= & a \cdot x(t)+\rho_{0}^{2} \exp \left(-\frac{2 p}{q} \phi(t)\right) \\
& \cdot \exp \left(2\left(b-\frac{p}{q} c\right) t\right) \cdot\left(b_{11} \cos ^{2} \phi(t)\right. \\
& \left.+b_{12} \cos \phi(t) \sin \phi(t)+b_{22} \sin ^{2} \phi(t)\right), \\
\dot{\phi}(t)= & -c-q \cdot x(t) .
\end{aligned}\right.
$$

Let us calculate Lyapunov's exponent $\Lambda$ for a real function $f(t)$ [Kuznetsov, 1998]:

$$
\Lambda[f]=\varlimsup_{t \rightarrow \infty} \frac{1}{t} \ln \left|\frac{f(t)}{f\left(t_{0}\right)}\right| .
$$

We take advantage of the following properties of Lyapunov's exponents:

(a1) $\Lambda\left[f_{1}(t) \cdot f_{2}(t)\right] \leq \Lambda\left[f_{1}(t)\right]+\Lambda\left[f_{2}(t)\right]$;

(a2) if $m \geq 0$, then $\Lambda\left[t^{m}\right]=0$;

(a3) if $\Lambda[f(t)]<0$, then $\Lambda\left[\int_{t}^{\infty} f(\tau) d \tau\right] \leq \Lambda[f(t)]$;

(a4) $\Lambda\left[f_{1}(t)+f_{2}(t)\right] \leq \max \left(\Lambda\left[f_{1}(t)\right], \Lambda\left[f_{2}(t)\right]\right)$;

(a5) $\Lambda[d \cdot f(t)]=\Lambda[f(t)](d \neq 0)$.

First we calculate $\Lambda[\rho]$. It is clear that $\max _{t>0} \rho(t)$ may be reached under the condition $\dot{\rho}(t)=0$ or at $x=-b / p$ [it follows from the second equation of system (12)]. In this case from the third equation of system (14) it follows that $\varlimsup_{t \rightarrow \infty} \rho(t) \rightarrow$ $\max$ if $\varlimsup_{t \rightarrow \infty} \dot{\phi}(t)=-c+b(q / p)$. Thus,

$$
\begin{aligned}
\Lambda[\rho] & =\varlimsup_{t \rightarrow \infty} \frac{1}{t} \ln \left|\frac{\rho(t)}{\rho_{0}}\right| \\
& =\varlimsup_{t \rightarrow \infty} \frac{1}{t} \ln \left|\frac{\rho_{0} \exp \left(-\frac{p}{q} \phi(t)+\left(b-\frac{p}{q} c\right) t\right)}{\rho_{0}}\right| \\
& =-\frac{p}{q} \varlimsup_{t \rightarrow \infty} \frac{\phi(t)}{t}+b-\frac{p}{q} c .
\end{aligned}
$$


Using L'Hospital's rule, we get

$$
\begin{aligned}
\Lambda[\rho] & =-\frac{p}{q} \varlimsup_{t \rightarrow \infty} \frac{\phi(t)}{t}+b-\frac{p}{q} c \\
& =-\frac{p}{q} \varlimsup_{t \rightarrow \infty} \dot{\phi}(t)+b-\frac{p}{q} c \\
& =0 .
\end{aligned}
$$

Consider the function

$$
\begin{aligned}
h(\phi)= & b_{11} \cos ^{2} \phi+b_{12} \cos \phi \sin \phi+b_{22} \sin ^{2} \phi \\
= & \frac{b_{11}+b_{22}}{2}+\frac{b_{11}-b_{22}}{2} \cos 2 \phi \\
& +\frac{b_{12}}{2} \sin 2 \phi \\
= & \frac{b_{11}+b_{22}}{2}+\frac{\sqrt{\left(b_{11}-b_{22}\right)^{2}+b_{12}^{2}}}{2} \\
& \times \sin (2 \phi+\alpha),
\end{aligned}
$$

where

$$
\begin{aligned}
& \sin \alpha=\frac{b_{11}-b_{22}}{\sqrt{\left(b_{11}-b_{22}\right)^{2}+b_{12}^{2}}}, \\
& \cos \alpha=\frac{b_{12}}{\sqrt{\left(b_{11}-b_{22}\right)^{2}+b_{12}^{2}}} .
\end{aligned}
$$

It is clear that $h(\phi) \neq 0$, if

$$
\frac{\left|b_{11}+b_{22}\right|}{\sqrt{\left(b_{11}-b_{22}\right)^{2}+b_{12}^{2}}}>1 \text {. }
$$

In this case for the function $h(\phi(t))$, Lyapunov's exponent $\Lambda(h)=0$. If

$$
\frac{\left|b_{11}+b_{22}\right|}{\sqrt{\left(b_{11}-b_{22}\right)^{2}+b_{12}^{2}}} \leq 1,
$$

then there exists a point $\phi=\gamma$ such that $h(\gamma)=0$. In this case $\Lambda(h)=-\infty$.

From here and (a1) it follows that $\Lambda\left(\exp (a t) \rho^{2} h\right) \leq \Lambda(\exp (a t))+\Lambda\left(\rho^{2}\right)+\Lambda(h) \leq$ $a+0+0=a$ or $\Lambda\left(\exp (a t) \rho^{2} h\right)=-\infty$. Thus, from (a2) and (a3) it follows that

$$
\Lambda\left[\int_{t}^{\infty} \exp (a(t-\tau)) \rho^{2}(\tau) h(\tau) d \tau\right] \leq a .
$$

Therefore, from boundedness of $x(t)$ and (a4), we have $\Lambda[x(t)] \leq a$.
From the third equation of system (12), we get $\phi(t)=\phi_{0}-c t+q \int_{0}^{t} x(\tau) d \tau$. Then from (a4) and (a5) it follows that

$$
\begin{aligned}
\Lambda[\phi(t)] & \leq \Lambda\left[\phi_{0}-c t\right]+\Lambda\left[q \int_{0}^{\infty} x(t) d t\right] \\
& \leq \max (0, a) \\
& =0 .
\end{aligned}
$$

As $a<0$, then $\Lambda[x]+\Lambda[\rho]+\Lambda[\phi] \leq a<0$. It means that system (12) [or (10)] is dissipative.

The origin is a unique equilibrium of system (10). If $b<0$ then the origin is a stable node. Therefore, by virtue of boundedness of solutions it will be attracted to the origin. If $b>0$, then the origin is a saddle-focus, and we have $\Lambda[x] \leq a<0$, $\Lambda[\rho]=0, \Lambda[\phi] \leq 0$.

(B) According to the conditions of Theorem 4, solutions $x(t)$ and $\rho(t)$ of system (12) are bounded at any initial data. Consequently for the concrete solution $\mathbf{x}\left(t, \mathbf{x}_{0}\right)$ (in polar coordinates it is $(x(t), \rho(t), \phi(t)))$ there is a nonempty, compact, invariant set $\mathbb{L}^{+}$(see Lemma 1 ).

Assume that a vector solution $\left(x^{+}(t), \rho^{+}(t)\right.$, $\left.\phi^{+}(t)\right) \in \mathbb{L}^{+}$. By virtue of boundedness of any solution $x(t), \rho(t)$ of system (12) there must be points $t_{k}$ such that $\dot{x}^{+}\left(t_{k}\right)=0 ; k=1,2, \ldots$. Therefore, from the first equation of system (12) and condition $\dot{x}^{+}\left(t_{k}\right)=0$, we have $h\left(\phi^{+}\left(t_{k}\right)\right)=$ $-a x^{+}\left(t_{k}\right) / \rho^{+2}\left(t_{k}\right)>0$.

We will consider that $x_{0}^{+}>0$. Then from this condition and the first equation of system (12) it follows that $\forall t>0 x(t)>0$ (see the proof of Theorem 3). As the function $h(\phi)$ is $\pi$-periodic and $h(\phi) \geq 0$, then the relation $x^{+}\left(t_{k}\right) / \rho^{+2}\left(t_{k}\right)$ is also $\pi$-periodic; $k=1,2, \ldots$.

There are only two possibilities:

(B1) The functions $x^{+}(t)$ and $\rho^{+}(t)$ are periodic;

(B2) The functions $x^{+}(t)$ and $\rho^{+}(t)$ are not periodic.

Assume that condition (B2) takes place. Then there does not exist a number $T>0$ such that $\forall t \geq 0 \rho^{+}(t+T)=\rho^{+}(t)$. Consequently the inequality

$$
\begin{aligned}
& -\frac{p}{q} \phi^{+}(t)+\left(b-\frac{p}{q} c\right) t \\
& \quad \neq-\frac{p}{q} \phi^{+}(t+T)+\left(b-\frac{p}{q} c\right)(t+T)
\end{aligned}
$$


or

$$
\frac{p}{q} \phi^{+}(t+T)-\frac{p}{q} \phi^{+}(t) \neq\left(b-\frac{p}{q} c\right) T
$$

follows from (13).

From here it follows that

$$
\begin{array}{r}
\frac{p}{q} \lim _{T \rightarrow 0} \frac{\phi^{+}(t+T)-\phi^{+}(t)}{T} \\
\quad=\frac{p}{q} \dot{\phi}^{+}(t) \neq b-\frac{p}{q} c .
\end{array}
$$

Thus, from the third equation of system (12) we have

$$
\forall t \geq 0 \quad \dot{\phi}(t)=-c-q x^{+}(t) \neq b \frac{q}{p}-c
$$

and

$$
x(t)^{+} \neq-\frac{b}{p}
$$

Then from the second equation of system (12) it follows that $\forall t \geq 0 \dot{\rho}^{+}(t) \neq 0$. It means that the function $\rho^{+}(t)$ is unbounded. We derived the contradiction. Consequently the condition (B1) must be valid.

In (A) for any solution $(x(t), \rho(t), \phi(t))$ the conditions $\Lambda[x] \leq a<0, \Lambda[\rho]=0, \Lambda[\phi] \leq 0$ were obtained. In addition, we know that the periodic solution $\left(x^{+}(t), \rho^{+}(t), \phi^{+}(t)\right)$ exists. From here it follows that in system (12) [or (10)] there is either a limit cycle $\mathbb{L}^{+}=\left(x^{+}(t), \rho^{+}(t), \phi^{+}(t)\right)$ or a limit torus (if $\Lambda[\phi]=0$ ).

Consider the following system

$$
\left\{\begin{array}{l}
\dot{x}(t)=a_{11} x(t)+h(\cos \phi(t), \sin \phi(t)) \rho^{2}(t), \\
\dot{\rho}(t)=a_{22} \rho(t)+c_{11} x(t) \rho(t), \\
\dot{\phi}(t)=-a_{23} .
\end{array}\right.
$$

This system is obtained from system (8) at values of parameters: $a_{12}=a_{13}=a_{21}=a_{31}=c_{12}=c_{21}=0$, $c_{11}=c_{22} \neq 0$, and $a_{23} \neq 0$. System (15) will be used for the construction of discrete models for system (7).

\section{Discrete Dynamics of System (15)}

Theorem 5. Let $a_{22}>0$. Then under the conditions of Theorem 4, a discrete dynamics of system (15) is determined by the following $2 D$ implicit iterated process:

$$
\left\{\begin{aligned}
x_{k+1}= & x_{k} \exp \left[\frac{2 \pi a_{22}}{a_{23}}+\frac{2 \pi c_{11}}{a_{23}}\right. \\
& \left.\times\left[u_{k+1} x_{k+1}-u_{k} x_{k}\right]\right], \\
u_{k+1}= & u_{k}+1 ; \quad k=0,1,2, \ldots
\end{aligned}\right.
$$

Proof

(A) In the beginning we will consider a system which is obtained from system (15) at the values of parameters $b_{12}=0$ and $b_{11}=b_{22}$ :

$$
\left\{\begin{array}{l}
\dot{x}(t)=a_{11} x(t)+b_{11} \rho^{2}(t), \\
\dot{\rho}(t)=a_{22} \rho(t)+c_{11} x(t) \rho(t) .
\end{array}\right.
$$

Let $x_{0}>0$ and $\rho_{0}>0$ be the initial values for system (17).

It is clear that system (17) has two equilibrium points, one of which is located in the first orthant:

$$
\begin{aligned}
& O_{1}=(0,0) ; \\
& O_{2}=\left(-\frac{a_{22}}{c_{11}}, \sqrt{\frac{a_{11} a_{22}}{c_{11} b_{11}}}\right) .
\end{aligned}
$$

It is possible to check that the point $O_{1}$ is saddle. At the point $O_{2}$, the Jacobian matrix of system (17) has eigenvalues

$$
\mu_{1,2}=\frac{a_{11}}{2} \pm \frac{\sqrt{\Delta}}{2}, \quad a_{11}<0
$$

where $\Delta=a_{11}^{2}+8 a_{11} a_{22}$. Hence, if $\Delta \geq 0$ then point $\mathrm{O}_{2}$ is a stable node; if $\Delta<0$ then point $\mathrm{O}_{2}$ is a stable focus.

Thus, in a small enough neighborhood of point $\mathrm{O}_{2}$ the solutions of system (17) look like:

(a) $\Delta \geq 0$

$$
\left\{\begin{aligned}
x(t)= & -\frac{a_{22}}{c_{11}}+\left(x_{0}+\frac{a_{22}}{c_{11}}\right) \\
& \times \exp \left(\left(0.5 a_{11}+0.5 \sqrt{\Delta}\right) t\right), \\
\rho(t)= & \sqrt{\frac{a_{11} a_{22}}{c_{11} b_{11}}}+\left(\rho_{0}-\sqrt{\frac{a_{11} a_{22}}{c_{11} b_{11}}}\right) \\
& \times \exp \left(\left(0.5 a_{11}-0.5 \sqrt{\Delta}\right) t\right) .
\end{aligned}\right.
$$


(b) $\Delta<0$,

$$
\left\{\begin{aligned}
x(t)= & -\frac{a_{22}}{c_{11}}+\exp \left(0.5 a_{11} t\right) \\
& \times\left[\left(x_{0}+\frac{a_{22}}{c_{11}}\right) \cos (0.5 \sqrt{-\Delta} t)\right. \\
& \left.-\left(\rho_{0}-\sqrt{\frac{a_{11} a_{22}}{c_{11} b_{11}}}\right) \sin (0.5 \sqrt{-\Delta} t)\right], \\
\rho(t)= & \sqrt{\frac{a_{11} a_{22}}{c_{11} b_{11}}}+\exp \left(0.5 a_{11} t\right) \\
& \times\left[\left(x_{0}+\frac{a_{22}}{c_{11}}\right) \sin (0.5 \sqrt{-\Delta} t)\right. \\
& \left.+\left(\rho_{0}-\sqrt{\frac{a_{11} a_{22}}{c_{11} b_{11}}}\right) \cos (0.5 \sqrt{-\Delta} t)\right] .
\end{aligned}\right.
$$

Introduce the designation

$$
u(t) \equiv \frac{\int_{0}^{t} x(\tau) d \tau}{x(t)} .
$$

We will consider an asymptotic behavior of function $u(t)$ at $t \rightarrow \infty$. From conditions $x_{0}>0$ and $\rho_{0}>0$, it follows that $x(t)>0$ and $\rho(t)>0$. Then the Bendixson Criterion [Kuznetsov, 1998] asserts that in the quadrant $x \geq 0, \rho \geq 0$ limit cycles do not exist. Consequently, any trajectory of system (17) will tend to the point $O_{2}$. Therefore, at $t \rightarrow \infty$, from (a) and (b) it follows that

$$
\begin{aligned}
& \lim _{(x(t), \rho(t)) \rightarrow O_{2}} u(t) \\
& =\lim _{(x(t), \rho(t)) \rightarrow O_{2}} \frac{\int_{0}^{t} x(\tau) d \tau}{x(t)} \\
& =\lim _{(x(t), \rho(t)) \rightarrow O_{2}} \frac{-\frac{a_{22} t}{c_{11}}+g_{0}+g_{1}(t) \exp \left(0.5 a_{11} t\right)}{-\frac{a_{22}}{c_{11}}+g_{2}(t) \exp \left(0.5 a_{11} t\right)}
\end{aligned}
$$$$
=t \text {. }
$$

(Here $g_{0}=$ const; $g_{1}(t)$ and $g_{2}(t)$ are bounded functions on interval $[0, \infty)$.)

Thus, at $t \rightarrow \infty$, we obtain the asymptotic estimates: $u(t)=t+O(1)$ and

$$
\int_{0}^{t} x(\tau) d \tau=-\frac{a_{22}}{c_{11}} t+O(1) .
$$

(B) Now we will consider that even one of the parameters $b_{12}$, or $b_{11}$, or $b_{22}$ is not equal to zero.

Let $x\left(t_{i}\right)=x_{i}, \rho\left(t_{i}\right)=\rho_{i}, \phi\left(t_{i}\right)=\phi_{i}$, where $t_{i}$ are roots of the first equation $\dot{x}\left(t_{i}\right)=a_{11} x\left(t_{i}\right)+$ $h\left(\cos \phi\left(t_{i}\right), \sin \phi\left(t_{i}\right)\right) \rho^{2}\left(t_{i}\right)=0$ of system (15), $i=$ $1,2, \ldots$ We can consider that $\cdots<\phi_{i-1}<\phi_{i}<$ $\phi_{i+1}<\phi_{i+2}<\cdots$.

We can also consider that the following variant takes place: $t_{k}, t_{k+1}, \ldots$ are sequential maximums of function $x(t) ; k=1,2, \ldots$.

From system (15) we have:

$$
\begin{array}{r}
x\left(t_{k}\right)=-\frac{\rho^{2}\left(t_{k}\right) \cdot h\left(\cos \phi\left(t_{k}\right), \sin \phi\left(t_{k}\right)\right)}{a_{11}}, \\
k=1,2, \ldots .
\end{array}
$$

According to Theorem 4, system (15) must have a limit cycle. Let $T$ be a period of this cycle. Then from (19) we have

$$
\begin{aligned}
\frac{x(t+T)}{\rho^{2}(t+T)} & =\frac{x(t)}{\rho^{2}(t)}=\frac{x\left(t_{k}\right)}{\rho^{2}\left(t_{k}\right)} \\
& =-\frac{h\left(\cos \phi\left(t_{k}\right), \sin \phi\left(t_{k}\right)\right)}{a_{11}} .
\end{aligned}
$$

Consider the fraction

$$
\frac{x_{k+1}}{x_{k}}=\frac{\rho_{k+1}^{2} \cdot h\left(\cos \phi\left(t_{k+1}\right), \sin \phi\left(t_{k+1}\right)\right)}{\rho_{k}^{2} \cdot h\left(\cos \phi\left(t_{k}\right), \sin \phi\left(t_{k}\right)\right)} .
$$

From (19) it follows that $\forall i$ the magnitude $\phi\left(t_{k+1}\right)-\phi\left(t_{k}\right)=\phi(T)$, where $\phi(T)$ is a period of the function $h(\cos \phi(t), \sin \phi(t))$. Then from here and (20), we have

$$
\frac{h\left(\cos \phi\left(t_{k+1}\right), \sin \phi\left(t_{k+1}\right)\right)}{h\left(\cos \phi\left(t_{k}\right), \sin \phi\left(t_{k}\right)\right)}=1 .
$$

It is clear that the period of the function $h(\cos \phi(t), \sin \phi(t))$ is equal to $\pi$. Therefore, period $T$ of the function $x(t)$ (or $\rho(t))$ is $n \pi$, where $n$ is an integer. Let $n=1$. Then from the third equation of system (15), we have

$$
\begin{aligned}
& \phi\left(t_{k+1}\right)-\phi\left(t_{k}\right) \\
& \quad=-\pi=-a_{23}\left(t_{k+1}-t_{k}\right) ; \quad a_{23}>0 .
\end{aligned}
$$

(If $a_{23}<0$, then $\phi\left(t_{k+1}\right)-\phi\left(t_{k}\right)=\pi$.)

Therefore, the fraction $x_{k+1} / x_{k}$ may be rewritten as

$$
\frac{x_{k+1}}{x_{k}}=\exp \left[\frac{2 \pi a_{22}}{a_{23}}+2 c_{11} \int_{t_{k}}^{t_{k+1}} x(\tau) d \tau\right] .
$$


We consider the sequence of systems of type (17):

$$
\begin{aligned}
& \left\{\begin{array}{l}
\dot{x}_{k}(t)=a_{11} x_{k}(t)+h\left(\cos \phi\left(t_{k}\right), \sin \phi\left(t_{k}\right)\right) \rho_{k}^{2}(t), \\
\dot{\rho}_{k}(t)=a_{22} \rho_{k}(t)+c_{11} x_{k}(t) \rho_{k}(t),
\end{array}\right. \\
& k=0,1,2, \ldots \text {. }
\end{aligned}
$$

Since $h\left(\cos \phi\left(t_{m}\right), \sin \phi\left(t_{m}\right)\right)=h\left(\cos \phi\left(t_{n}\right)\right.$, $\left.\sin \phi\left(t_{n}\right)\right)$ for any $m, n=0,1,2, \ldots$, it is possible to consider that $b_{11}=h\left(\cos \phi\left(t_{m}\right), \sin \phi\left(t_{m}\right)\right)>0$, $m=0,1,2, \ldots$ Therefore, for any $t_{0}, t_{1}, \ldots$ the equilibrium points of system (22) coincide with points $O_{1}, O_{2}$ having the type: a saddle and a stable node or a stable focus. Thus, in a small enough neighborhood of point $O_{2}$ for any $k=0,1,2, \ldots$, we obtain the same solutions (a) and (b) of system (22) that in the section (A): $x_{k}(t)=x(t)$ and $\rho_{k}(t)=\rho(t)$.

Rewrite relation (18) in the form

$$
\int_{0}^{t} x(\tau) d \tau=x(t) u(t)
$$

where $u(t)$ is differentiated on the interval $[0, \infty)$ function.

Differentiate relation (23) on $t$. Then taking into account (15), we derive

$\dot{u}(t)=-u(t)\left[a_{11}+h(\cos \phi(t), \sin \phi(t)) \frac{\rho^{2}(t)}{x(t)}\right]+1$.

Introduce the designation

$$
f(t) \equiv-\left[a_{11}+h(\cos \phi(t), \sin \phi(t)) \frac{\rho^{2}(t)}{x(t)}\right]
$$

where $f\left(t_{k}\right)=-\dot{x}\left(t_{k}\right) / x\left(t_{k}\right)=0 ; k=0,1, \ldots$ Then we will have

$$
\dot{u}(t)=f(t) u(t)+1 \text {. }
$$

Let $\xi$ be any fixed point of the increasing sequence $t_{0}, t_{1}, t_{2}, \ldots ; \lim _{k \rightarrow \infty} t_{k}=\infty$. (In other words, $f(\xi)=0$.)

We choose a small enough positive interval $(\xi-\delta, \xi+\delta)$ containing the point $\xi$. (Here $\delta \rightarrow 0$.)

As $x(t)=-a_{22} / c_{11}+o(1)$, then $f(t)=o(1)$ at $t \rightarrow \infty$. Let $f_{\max }=\max _{t}|f(t)|, t \in[\xi-\delta, \xi+\delta]$. Instead of Eq. (24), we consider equation

$$
\dot{v}(t)=f_{\max } v(t)+1 .
$$

(It is clear that $\forall t \in(\xi-\delta, \xi+\delta) u(t) \leq v(t)$.)
The solution of the linear equation (25) at $t \rightarrow \xi$ has the form

$$
v(t)=\frac{v_{0} \exp \left(\int_{0}^{t} f_{\max } d \tau\right)-1}{f_{\max }} .
$$

The Taylor-series expansion of the exponent in a neighborhood of the point $t=\xi$ gives such a result:

$$
\begin{aligned}
v(t)= & \lim _{\tau \rightarrow \xi} \frac{v_{0}+v_{0} f_{\max }(t-\xi)+\cdots-1}{f_{\max }} \\
= & \frac{v_{0}-1}{f_{\max }}+v_{0}(t-\xi)+\lim _{\tau \rightarrow \xi} \frac{v_{0}}{f_{\max }} \\
& \times \sum_{i=1}^{\infty} \frac{1}{(i+1) !} f_{\max }^{i}(\tau-\xi)^{i+1} \\
= & v_{0}(t-\xi)+\frac{v_{0}-1}{f_{\max }}+o(1) .
\end{aligned}
$$

As $v(t)$ does not depend on $\delta$, then $\lim _{\delta \rightarrow \xi} v(t)=\beta(t-\xi)+\left(u_{0}-1\right) / f_{\max }=\beta t+\alpha ;$ $\alpha=$ const, $\beta=$ const. The last relation results in the formula

$$
\begin{aligned}
v_{k+1}-v_{k} & =\beta t_{k+1}+\alpha-\beta t_{k}-\alpha \\
& =\beta t_{k+1}-\beta t_{k}=\beta \frac{\pi}{a_{23}} .
\end{aligned}
$$
form

With regard to (23) equality (21) adopts the

$$
\frac{x_{k+1}}{x_{k}}=\exp \left[\frac{2 \pi a_{22}}{a_{23}}+2 c_{11}\left(x_{k+1} v_{k+1}-x_{k} v_{k}\right)\right] .
$$

In order that the process (27) tends to the equilibrium point $\mathrm{O}_{2}$ it is necessary that $\lim _{k \rightarrow \infty} x_{k}=$ $-a_{22} / c_{11}$ and $v_{0}=1$. From here and (26) it follows that $\beta=1$.

Fulfill the replacement of variables: $v \rightarrow \pi u /$ $a_{23}$. Now a union of Eqs. (27) and (26) completes the proof of Theorem 5 .

The following obvious assertion is derived by the calculation of a fixed point of map (16).

Corollary. Under the conditions of Theorem 5 system (15) has a limit cycle which is a circumference

$$
y^{2}+z^{2}=\frac{a_{11} a_{22}}{c_{11} b_{11}}
$$

situated in the plane $x=-a_{22} / c_{11}$. 
Notice that the value $u$ substantially depends on the initial data. Therefore, for a hit on the limit cycle, it is necessary that time $t$ was large enough.

Assume that in systems (7) and (8): $a_{11}=a$, $a_{12}=a_{13}=0, c_{12}=c_{21}=0, c_{11}=c_{22}=p, a_{22}=b$, $a_{23}=c$,

$$
s=\frac{b_{11}+b_{22}}{2}, \quad d= \pm \frac{\sqrt{\left(b_{11}-b_{22}\right)^{2}+b_{12}^{2}}}{2} .
$$

Then we have

$$
\left\{\begin{array}{l}
\dot{x}(t)=a x(t)+b_{11} y^{2}(t)+b_{22} z^{2}(t), \\
\dot{y}(t)=a_{21} x(t)+b y(t)+c z(t)+p x(t) y(t), \\
\dot{z}(t)=a_{31} x(t)-c y(t)+b z(t)+p x(t) z(t)
\end{array}\right.
$$

and

$$
\left\{\begin{array}{l}
\dot{x}(t)=a x+(s+d \cdot \sin (2 \phi+\alpha)) \rho^{2}, \\
\dot{\rho}(t)=\left(a_{21} \cos \phi+a_{31} \sin \phi\right) x+b \rho+p x \rho, \\
\dot{\phi}(t)=-c+\left(a_{31} \cos \phi-a_{21} \sin \phi\right) \frac{x}{\rho} .
\end{array}\right.
$$

Here $a<0, b>0, s>0, s-|d|>0, p<0$, and the angle $\alpha$ is defined in Sec. 3 .

System (29) can be rewritten as following

$$
\left\{\begin{array}{l}
\dot{x}(t)=a x+(s+d \cdot \sin (2 \phi+\alpha)) \rho^{2}, \\
\dot{\rho}(t)=q \cdot(\cos (\phi+\beta)) x+b \rho+p x \rho, \\
\dot{\phi}(t)=-c-q \cdot(\sin (\phi+\beta)) \frac{x}{\rho}
\end{array}\right.
$$

where

$$
\begin{gathered}
q=\sqrt{a_{21}^{2}+a_{31}^{2}}, \quad \sin \beta=-\frac{a_{31}}{\sqrt{a_{21}^{2}+a_{31}^{2}}}, \\
\cos \beta=\frac{a_{21}}{\sqrt{a_{21}^{2}+a_{31}^{2}}} .
\end{gathered}
$$

Introduce a new variable under the formula $\xi(t)=\phi(t)+\beta$. Then system (30) transforms to the system

$$
\left\{\begin{array}{l}
\dot{x}(t)=a x+(s+d \cdot \sin (2 \xi+\alpha-2 \beta)) \rho^{2}, \\
\dot{\rho}(t)=q \cdot(\cos (\xi)) x+b \rho+p x \rho \\
\dot{\xi}(t)=-c-q \cdot(\sin (\xi)) \frac{x}{\rho}
\end{array}\right.
$$

Below we will consider the simplified variant (31) of system (8).

\section{Chaotic Dynamics in System (31) and Lambert Function}

There are different methods to establish the presence of chaotic dynamics in the nonlinear systems. We will adhere to the following approach: at first $1 \mathrm{D}$ discrete map $P: \mathbb{R} \rightarrow \mathbb{R}$ which can generate chaos in system (31) is built; after that its chaoticity (in the sense of both Li-Yorke and Devaney) is proved [Belozyorov, 2012; Belozyorov \& Chernyshenko, 2013].

System (31) is called chaotic if the map $P$ is chaotic.

In [Belozyorov, 2011b] the discrete map

$$
\begin{array}{r}
z_{k+1}=z_{k} \cdot \exp \left(\lambda-\alpha_{2 k} z_{k}-\alpha_{2 k+1} z_{k+1}\right) ; \\
k=0,1,2, \ldots,
\end{array}
$$

describing a chaotic dynamics in systems (7) [or (8)] at $a_{21}=a_{31}=0$ was obtained. (Here $\lambda, \alpha_{0}$, $\alpha_{1}, \ldots$ are known positive real numbers.)

For system (15), Theorem 4 gives a more general result. The point $x_{k}$ of process (16) depends only on the parameters of system (15). At the same time, process (32) depends not only on the parameters of system (7), but also on the character of its solutions. (This character is included in the sequence of coefficients $\alpha_{0}, \alpha_{1}, \ldots$ of process (32).) Seems that in the case of system (7) [or (8)] to get a simple discrete map of type (32) is already impossible. Nevertheless for research of chaos in system (8), we have applied the same approach as for the construction of map (32) [Belozyorov, 2011b].

The solution of the second equation of system (31) can be represented in the form

$$
\begin{aligned}
\rho(t)= & \rho_{0} \exp \left[\int_{0}^{t}(b+p x(\tau)) d \tau\right] \\
& +q \int_{0}^{t} x(\tau) \cos (\xi(\tau)) \\
& \times \exp \left[\int_{\tau}^{t}(b+p x(\tau)) d \tau\right] d \tau \\
= & \exp \left[\int_{0}^{t}(b+p x(\omega)) d \omega\right] \\
& \times\left[\rho_{0}+q \int_{0}^{t} x(\tau) \cos (\xi(\tau))\right. \\
& \left.\times \exp \left[-\int_{0}^{\tau}(b+p x(\tau)) d \tau\right] d \tau\right], \quad t>\tau .
\end{aligned}
$$


Suppose that $\forall \rho_{0}>0, x_{0}>0, t \geq 0$ and $\forall q \in \mathbb{R}$, the condition

$$
\forall t \geq 0 \quad \rho(t)>0
$$

is valid.

Let $m$ be a positive integer. With the help of formula (32), we define the real implicit 1D iterated process by the rule

$$
\begin{aligned}
z_{k+m}= & z_{k} \cdot \exp \left[m \cdot \lambda-\alpha_{2 k} z_{k}\right. \\
& -\left(\alpha_{2 k+1}+\alpha_{2 k+2}\right) z_{k+1} \\
& -\cdots-\left(\alpha_{2 k+2 m-3}+\alpha_{2 k+2 m-2}\right) z_{k+m-1} \\
& \left.-\alpha_{2 k+2 m-1} z_{k+m}\right] ; \quad k=0,1, \ldots
\end{aligned}
$$

Here $\lambda, \alpha_{0}, \alpha_{1}, \ldots$ are the same numbers as that in $(32)$.

Consider the discrete 1D map

$$
\begin{array}{r}
x_{k+1}=x_{k} \cdot \exp \left(\lambda-\alpha_{0} x_{k}-\alpha_{1} x_{k+1}\right), \\
k=0,1,2, \ldots
\end{array}
$$

Lemma 2. If $\alpha_{0}=\alpha_{1}$ then map (36) is not chaotic.

Proof. Let $\alpha_{0}=\alpha_{1}=\alpha$. Then by replacement of variable $\alpha x \rightarrow y$ map (36) can be reduced to the form

$$
y_{k+1}=y_{k} \cdot \exp \left(\lambda-y_{k}-y_{k+1}\right), \quad k=0,1,2, \ldots
$$

It is clear that the point $y^{*}=\lambda / 2$ is a fixed point for map (37).
The next fixed point is determined from relation $y_{k+2}=y_{k+1} \cdot \exp \left(\lambda-y_{k+1}-y_{k+2}\right)$, where $y_{k+1} \neq y_{k} ; k=0,1,2, \ldots$

From the last formula and (37) it follows that $y_{k+2}=y_{k} \cdot \exp \left(2 \lambda-y_{k}-y_{k+2}-2 y_{k+1}\right)$.

Let $y_{k+2}=y_{k}$. Then we have $\lambda=y_{k}+y_{k+1}$ and from here and (37) it follows that $y_{k+1}=y_{k}$. We get the contradiction. Consequently, all iterations of map (37) has a unique fixed point $y^{*}=\lambda / 2$. Therefore (37) cannot be chaotic.

Theorem 6. Let the point $(0,0,0)$ be a unique equilibrium of system (28). Suppose also that for system (31) conditions $a<0, b>0, s>0, s-|d|>0$, $d \neq 0, p<0, c \neq 0, q \neq 0$, and (34) are valid. Then at some values of parameters $\lambda(q), \alpha_{0}(q)$, and $\alpha_{1}(q)$ a dynamics of system (31) defined by process (36) becomes chaotic.

Proof. The conditions $a<0, b>0, s>0$, $s-|d|>0, d \neq 0, p<0$, and $c \neq 0$ are the corollaries of Theorems 3 and 4 applied to system (31). Thus, the solutions $x(t)$ and $\rho(t)$ of system (31) are bounded.

Suppose that in formula (33) the variable $t$ takes two values: $t_{k}$ and $t_{k+1}$. Then we define the numbers $\rho\left(t_{k}\right)=\rho_{k}>0$ and $\rho\left(t_{k+1}\right)=\rho_{k+1}>0$. Introduce the designation

$$
\Delta(t)=\exp \left[\int_{t_{0}}^{t}(b+p x(\omega)) d \omega\right] .
$$

Then from formula (33) it follows that

$$
\begin{aligned}
\rho_{k+1} & =\rho_{0} \Delta\left(t_{k+1}\right)+q \Delta\left(t_{k+1}\right) \int_{t_{0}}^{t_{k+1}} \Delta(-\tau) x(\tau)(\cos \xi(\tau)) d \tau, \\
\rho_{k} & =\rho_{0} \Delta\left(t_{k}\right)+q \Delta\left(t_{k}\right) \int_{t_{0}}^{t_{k}} \Delta(-\tau) x(\tau)(\cos \xi(\tau)) d \tau
\end{aligned}
$$

and

$$
\begin{aligned}
\frac{\rho_{k+1}}{\rho_{k}}= & \Delta\left(t_{k+1}\right) \Delta\left(-t_{k}\right)+q \frac{\Delta\left(t_{k+1}\right)}{\rho_{k}} \int_{t_{k}}^{t_{k+1}} \Delta(-\tau) x(\tau)(\cos \xi(\tau)) d \tau \\
= & \Delta\left(t_{k+1}\right) \Delta\left(-t_{k}\right)+\frac{q \Delta\left(t_{k+1}\right) \Delta\left(-t_{k}\right)}{\rho_{0}+q \int_{t_{0}}^{t_{k}} \Delta(-\tau) x(\tau)(\cos \xi(\tau)) d \tau} \\
& \times \int_{t_{k}}^{t_{k+1}} \Delta(-\tau) x(\tau)(\cos \xi(\tau)) d \tau .
\end{aligned}
$$

Then according to the method in Sec. 3 from the last relation, we get that for any integer $k$ takes the following form 


$$
\begin{aligned}
\frac{x_{k+1}}{x_{k}}= & {\left[\frac{\rho_{k+1}}{\rho_{k}}\right]^{2}=\exp \left[2 \int_{t_{k}}^{t_{k+1}}(b+p x(\omega)) d \omega\right] } \\
& \times\left[\begin{array}{c}
\left.1+\frac{q \int_{t_{k}}^{t_{k+1}} x(\tau)(\cos \xi(\tau)) \exp \left[-\int_{t_{k}}^{t_{k}+\tau}(b+p x(\tau)) d \tau\right] d \tau}{\left.\rho_{0}+q \sum_{i=0}^{k-1} \int_{t_{i}}^{t_{i+1}} x(\tau)(\cos \xi(\tau)) \exp \left[-\int_{t_{i}}^{t_{i}+\tau}(b+p x(\tau)) d \tau\right] d \tau\right]}\right]^{2} ; t_{k+1} \geq t_{k}+\tau>t_{k} .
\end{array}\right.
\end{aligned}
$$

Consider the series $(k \rightarrow \infty)$ located in a denominator of expression (38):

$$
\rho_{0}+q \sum_{i=0}^{\infty} \int_{t_{i}}^{t_{i+1}} x(\tau)(\cos \xi(\tau)) \exp \left[-\int_{t_{i}}^{t_{i}+\tau}(b+p x(\tau)) d \tau\right] d \tau
$$

(A) Assume that series (39) converges and it has a finite sum; this sum is denoted by

$$
S=\rho_{0}+\lim _{k \rightarrow \infty} S_{k}>0
$$

where $S_{k}$ is a $k$ th partial sum of series (39). Then there exists a constant $K>0$ such that

$$
0<\exp \left[-\int_{t_{k}}^{t_{k}+\tau}(b+p x(\tau)) d \tau\right] \leq K, \quad t_{k}<\tau<t_{k+1} .
$$

By virtue of convergence of series (39) the following condition takes place:

$$
\lim _{k \rightarrow \infty} \exp \left[-\int_{t_{k}}^{t_{k}+\tau}(b+p x(\tau)) d \tau\right]=0 .
$$

Take advantage of equality (41). Then from (38) we have the following chain of transformations:

$$
\begin{gathered}
\lim _{k \rightarrow \infty}\left[1+\frac{q \int_{t_{k}}^{t_{k+1}} x(\tau)(\cos \xi(\tau)) \exp \left[-\int_{t_{k}}^{t_{k}+\tau}(b+p x(\tau)) d \tau\right] d \tau}{\left.\rho_{0}+q \sum_{i=0}^{k-1} \int_{t_{i}}^{t_{i+1}} x(\tau)(\cos \xi(\tau)) \exp \left[-\int_{t_{i}}^{t_{i}+\tau}(b+p x(\tau)) d \tau\right] d \tau\right]}\right] \\
=1+q \lim _{k \rightarrow \infty} \frac{\exp \left[-\int_{t_{k}+\nu_{k}}^{t_{k}+\mu_{k}}(b+p x(\tau)) d \tau\right] \int_{t_{k}}^{t_{k+1}} x(\tau) \cos \xi(\tau) d \tau}{S}=1 .
\end{gathered}
$$

Here $\mu_{k}>0, \nu_{k}>0, t_{k} \leq t_{k}+\nu_{k}<t_{k}+\mu_{k} \leq t_{k+1}$.

(B) Now let conditions (41) and $S=0$ be valid. Then $\rho_{0}+\lim _{k \rightarrow \infty} S_{k}=0$,

$$
\rho_{0}+q \sum_{i=0}^{\infty} \int_{t_{i}}^{t_{i+1}} x(\tau)(\cos \xi(\tau)) \exp \left[-\int_{t_{i}}^{t_{i}+\tau}(b+p x(\tau)) d \tau\right] d \tau=0
$$

and

$$
S-S_{k}-\rho_{0}=R_{k}=q \sum_{i=k}^{\infty} \int_{t_{i}}^{t_{i+1}} x(\tau)(\cos \xi(\tau)) \exp \left[-\int_{t_{i}}^{t_{i}+\tau}(b+p x(\tau)) d \tau\right] d \tau,
$$

where $R_{k}$ is a residual of series (39). In addition, $R_{k} \rightarrow 0$ as $k \rightarrow \infty$. 
Introduce the designation

$$
\begin{aligned}
F & =\lim _{k \rightarrow \infty}\left[1+\frac{q \int_{t_{k}}^{t_{k+1}} x(\tau)(\cos \xi(\tau)) \exp \left[-\int_{t_{k}}^{t_{k}+\tau}(b+p x(\tau)) d \tau\right] d \tau}{\left.\rho_{0}+q \sum_{i=0}^{k-1} \int_{t_{i}}^{t_{i+1}} x(\tau)(\cos \xi(\tau)) \exp \left[-\int_{t_{i}}^{t_{i}+\tau}(b+p x(\tau)) d \tau\right] d \tau\right]}\right]=\left(1+\frac{0}{0}\right) \\
& =\lim _{k \rightarrow \infty}\left[1+\frac{q \int_{t_{k}}^{t_{k+1}} x(\tau)(\cos \xi(\tau)) \exp \left[-\int_{t_{k}}^{t_{k}+\tau}(b+p x(\tau)) d \tau\right] d \tau}{\left.q \sum_{i=k}^{\infty} \int_{t_{i}}^{t_{i+1}} x(\tau)(\cos \xi(\tau)) \exp \left[-\int_{t_{i}}^{t_{i}+\tau}(b+p x(\tau)) d \tau\right] d \tau\right]}\right]
\end{aligned}
$$

Applying the L'Hospital's rule to the fraction located in large square brackets of formula (42) we get

$$
F=\lim _{k \rightarrow \infty}\left[1+\frac{\exp \left[-\int_{t_{k}}^{t_{k}+\tau}(b+p x(\tau)) d \tau\right] d \tau}{\left.\sum_{i=k}^{\infty} \exp \left[-\int_{t_{i}}^{t_{i}+\tau}(b+p x(\tau)) d \tau\right] d \tau\right]}\right]
$$

Introduce the designation $T=\max _{k}\left(t_{k+1}-t_{k}\right)>0$. (By virtue of construction of the sequence $t_{0}, t_{1}, \ldots, t_{k}, \ldots$ the sequence $t_{1}-t_{0}, t_{2}-t_{1}, \ldots, t_{k+1}-t_{k}, \ldots$ is uniformly bounded.) Then, by virtue of the boundedness of the positive function $x(t)$, for $i \geq k$ we have estimations

$$
\begin{aligned}
\exp \left[-\int_{t_{i}}^{t_{i}+\tau}(b+p x(\tau)) d \tau\right] d \tau & \geq \exp \left[-\int_{t_{i}}^{t_{i}+\tau}|b+p x(\tau)| d \tau\right] \geq \exp \left[-\int_{t_{i}}^{t_{i+1}}|b+p x(\tau)| d \tau\right] \\
& \geq \exp \left[-\int_{t_{k}}^{t_{i+1}}|b+p x(\tau)| d \tau\right] \geq \exp \left[-(i-k) \int_{t_{k}}^{t_{k+1}}|b+p x(\tau)| d \tau\right] .
\end{aligned}
$$

Suppose that the moments $t_{r}$ and $t_{r+1}$ from sequence $t_{0}, t_{1}, \ldots$ such that $T=t_{r+1}-t_{r}$.

We have $F>0$. On the other hand from (43), it follows that

$$
\begin{array}{r}
F \leq \lim _{k \rightarrow \infty}\left[1+\frac{\exp \left[-\int_{t_{k}}^{t_{k}+\tau}(b+p x(\tau)) d \tau\right] d \tau}{\left.\sum_{i=k+1}^{\infty} \exp \left[-(i-k) \int_{t_{i}}^{t_{i}+\tau}(b+p x(\tau)) d \tau\right] d \tau\right]}\right] \\
\leq \lim _{k \rightarrow \infty}\left[1+\frac{\exp \left[-\int_{t_{k}}^{t_{k+1}}|b+p x(\tau)| d \tau\right] d \tau}{\left.\sum_{i=k+1}^{\infty} \exp \left[-(i-k) \int_{t_{k}}^{t_{k+1}}|b+p x(\tau)| d \tau\right] d \tau\right]}\right] \\
\leq \lim _{k \rightarrow \infty}\left[1+\frac{\exp \left[-\int_{t_{r}}^{t_{r+1}}|b+p x(\tau)| d \tau\right] d \tau}{\sum_{i=k+1}^{\infty} \exp \left[-(i-k) \int_{t_{r}}^{t_{r+1}}|b+p x(\tau)| d \tau\right] d \tau}\right] .
\end{array}
$$


The denominator of the last formula is a decreasing geometrical progression $1+\theta+\theta^{2}+\cdots$, where for $q \neq 0$ we have

$$
\theta=\exp \left[-\int_{t_{r}}^{t_{r+1}}|b+p x(\omega)| d \omega\right], \quad \theta<1 .
$$

Hence,

$$
\begin{aligned}
F & \leq 1+\left[1-\exp \left[-\int_{t_{r}}^{t_{r+1}}|b+p x(\omega)| d \omega\right]\right] \\
& \equiv 1+\varepsilon(q)<2
\end{aligned}
$$

where the function

$$
\varepsilon(q)=1-\exp \left[-\int_{t_{r}}^{t_{r+1}}|b+p x(\omega)| d \omega\right]
$$

is defined for $q \neq 0$.

Thus, we obtain $0 \leq \varepsilon(q)<1$, and from (38) it follows that

$$
\begin{aligned}
\frac{x_{k+1}}{x_{k}}< & \exp \left[2 \int_{t_{k}}^{t_{k+1}}(b+p x(\omega)) d \omega\right] \\
\cdot & {\left[2-\exp \left[-\int_{t_{r}}^{t_{r+1}}|b+p x(\omega)| d \omega\right]\right]^{2} . }
\end{aligned}
$$

Now let only condition (40) be valid. Then series (39) diverges and the partial sums are uniformly bounded. Thus, from (38) we obtain

$$
\frac{x_{k+1}}{x_{k}}=\exp \left[2 \int_{t_{k}}^{t_{k+1}}(b+p x(\omega)) d \omega\right] \cdot(1+\varepsilon(q))^{2},
$$

where $0 \leq \varepsilon(q)<1$ and $\varepsilon(q)=0$ is valid only at $q=0$.

There is another possibility. Series (39) diverges and the partial sums are uniformly unbounded. In this case, if only one from inequalities (40) or (41) is fulfilled, then $\varepsilon(q)=0$. Otherwise we again get $\varepsilon(q) \neq 0$.

Let $t_{i, i+1} \in\left[t_{i}, t_{i+1}\right]$ be a minimum point bounded on $\left[t_{i}, t_{i+1}\right]$ function $x(t)$. The function $x(t)$ is monotone decreasing on interval $\left[t_{i}, t_{i, i+1}\right]$, and it is monotone increasing on interval $\left[t_{i, i+1}, t_{i+1}\right]$. Then for any continuous function $w(t)$, we have (the second theorem is known on average value):

$$
\begin{array}{rl}
\int_{t_{i}}^{t_{i+1}} & w(\tau) \cdot x(\tau) d \tau \\
\quad= & \int_{t_{i}}^{t_{i, i+1}} w(\tau) \cdot x(\tau) d \tau+\int_{t_{i, i+1}}^{t_{i+1}} w(\tau) \cdot x(\tau) d \tau
\end{array}
$$

$$
\begin{aligned}
& =x\left(t_{i}+0\right) \int_{t_{i}}^{\xi_{i}} w(\tau) d \tau+x\left(t_{i+1}-0\right) \\
& \quad \times \int_{\zeta_{i}}^{t_{i+1}} w(\tau) d \tau \\
& =\alpha_{2 i} x_{i}+\alpha_{2 i+1} x_{i+1},
\end{aligned}
$$

where $t_{i} \leq \xi_{i} \leq t_{i, i+1}, t_{i, i+1} \leq \zeta_{i} \leq t_{i+1} ; \alpha_{2 i}=$ $\int_{t_{i}}^{\xi_{i}} w(\tau) d \tau, \alpha_{2 i+1}=\int_{\zeta_{i}}^{t_{i+1}} w(\tau) d \tau$.

As $0 \leq \varepsilon(q)<1$ then the designation $\eta=$ $2 \ln (1+\varepsilon(q))$ is correct. Thus, we have

$$
\begin{aligned}
\exp & {\left[\eta+2 \int_{t_{k}}^{t_{k+1}}(b+p x(\omega)) d \omega\right] } \\
& =\exp \left[\eta+2 b\left(t_{k+1}-t_{k}\right)+2 p \int_{t_{k}}^{t_{k+1}} x(\omega) d \omega\right] \\
& =\exp \left(\lambda-\alpha_{2 k} x_{k}-\alpha_{2 k+1} x_{k+1}\right),
\end{aligned}
$$

where

$$
\begin{gathered}
\lambda=\eta+2 b\left(t_{k+1}-t_{k}\right), \\
\alpha_{2 k}=\frac{2 p \int_{t_{k}}^{t_{k, k+1}} x(\tau) d \tau}{x_{k}}, \\
\alpha_{2 k+1}=\frac{2 p \int_{t_{k, k+1}}^{t_{k+1}} x(\tau) d \tau}{x_{k+1}} ;
\end{gathered}
$$

$t_{k, k+1}$ is the point of minimum of the function $x(t)$ located between two sequential maximums of that function.

A cascade of bifurcations of a continuous 3D system begins from the bifurcation of a limit cycle. For the discrete 1D maps, this means the bifurcation of 1-cycle. Consequently, the 3D system has to contain a limit cycle.

Suppose that

$$
\lim _{k \rightarrow \infty} \alpha_{2 k}=\alpha_{0}, \quad \lim _{k \rightarrow \infty} \alpha_{2 k+1}=\alpha_{1} .
$$

It means that process (32) has $l$-cycle (integer $l \geq 1$ ) and system (31) has a limit cycle (it is a periodic solution).

Taking into account the process (32), it is possible to rewrite it in the form (36). (The coefficients $\alpha_{0}$ and $\alpha_{1}$ do not depend on $k$.)

In [Belozyorov, 2012] it is shown that the dynamics of process (36) at $q=0$ and some $\lambda>$ $0, \alpha_{0}>0$, and $\alpha_{1}>0$ become chaotic. (In accord with Lemma $2, \alpha_{0} \neq \alpha_{1}$.) This means that at some small enough values of $q$, process (36) will also 
remain chaotic. Finally, the uniqueness of the saddle point $(0,0,0)$ eliminates the possibility of the limit equalities $\lim _{t \rightarrow \infty} \dot{\xi}(t)=0$ and $\lim _{t \rightarrow \infty} \xi(t)=$ const. Hence we have $\lim _{k \rightarrow \infty} t_{k}=\infty$. Then from boundedness of $x(t), \rho(t) \neq 0$, and third equation of system (31), it follows that $\lim _{k \rightarrow \infty} \xi\left(t_{k}\right)=-\infty$, the equation $\sin \left(2 \xi\left(t_{k}\right)+\alpha-2 \beta\right)=m(|m| \leq 1)$ have solutions $\xi\left(t_{k}\right)$, and process (36) takes place. This completes the proof of Theorem 6 .

We notice that if in (31) $q=0$, then in (32), (35) and (36), $\lambda=2 \pi b / c$. In the case $q \neq 0$, we have generally speaking $\lambda \approx 2 \pi b / c$.

Let $q=0$. By virtue of (31) periodic solutions of the system have period $\pi$. If $q>0$ then solutions become already $k \pi$-periodic, where an integer $k>1$. Therefore, for the description of discrete processes in system (31) for $q>0$, it is necessary to use formula (35) at $m \geq 2$.

We remind that at $q=0$ solutions of system (31) have period $\pi$ and its discrete dynamics is described by the map (36). If $0<q<q^{*}$, where $q^{*}$ is a real number, a bifurcation of the limit cycle of system (31) takes place and its period becomes equal $2 \pi$. The discrete dynamics of system (31) will be already described by two consequent iterations:

$$
x_{k+1}=x_{k} \cdot \exp \left(\lambda_{0}-\alpha_{0} x_{k}-\alpha_{1} x_{k+1}\right)
$$

and

$$
\begin{array}{r}
x_{k+2}=x_{k+1} \cdot \exp \left(\lambda_{1}-\beta_{1} x_{k+1}-\beta_{2} x_{k+2}\right) ; \\
k=0,1,2, \ldots
\end{array}
$$

Eliminating from the last formula the variable $x_{k+1}$, we get

$$
\begin{aligned}
x_{k+2}= & x_{k} \cdot \exp \left(\lambda_{0}+\lambda_{1}-\alpha_{0} x_{k}-\beta_{2} x_{k+2}\right. \\
& \left.-\left(\alpha_{1}+\beta_{1}\right) \cdot \Theta\left(x_{k}, x_{k+2}\right)\right) ; \quad k=0,1,2, \ldots,
\end{aligned}
$$

where

$$
\begin{aligned}
\Theta\left(x_{k}, x_{k+2}\right)= & {\left[x _ { k } ^ { \beta _ { 1 } } x _ { k + 2 } ^ { \alpha _ { 1 } } \operatorname { e x p } \left(\lambda_{0} \beta_{1}-\lambda_{1} \alpha_{1}\right.\right.} \\
& \left.\left.-\beta_{1} \alpha_{0} x_{k}+\alpha_{1} \beta_{2} x_{k+2}\right)\right]^{\frac{1}{\alpha_{1}+\beta_{1}}} .
\end{aligned}
$$

(We note the occurrence so that $\alpha_{1}=\beta_{1}=0$. Then map (45) must be changed by the formula $x_{k+2}=$ $x_{k} \cdot \exp \left(\lambda_{0}+\lambda_{1}-\alpha_{0} x_{k}-\beta_{2} x_{k+2}\right)$.)

A further scenario is obvious: if $q^{*}<q<q^{* *}$, where $q^{* *}$ is a real number, the next doubling of period will be described by four processes (36) and so on. In conclusion, we notice that all parameters $\lambda_{0}, \alpha_{0}, \alpha_{1}, \ldots, \beta_{2}$ implicitly depend on $q$.
Equation (45) has a very composite structure. In connection with this remark, we introduce new concepts and denotations.

We remind that a function $y=y(x)$ is called the Lambert function if the following conditions hold: $\forall x \in[0, \infty) y(x) \cdot \exp (y(x)) \equiv x$. By $\operatorname{Lam}(x)$ denote the function $y(x)$. Thus, $y(x)=\operatorname{Lam}(x)$.

Now we replace the variable $x$ in Eq. (36) by the variable $\alpha_{1} z$. Then we have $z_{n+1} \exp z_{n+1}=$ $z_{n} \exp \left(\lambda-\mu z_{n}\right)$, where $\mu=\alpha_{0} / \alpha_{1}$. The use of the Lambert function results in relation

$$
z_{n+1}=\operatorname{Lam}\left(z_{n} \exp \left(\lambda-\mu z_{n}\right)\right), \quad n=0,1,2, \ldots
$$

This is an explicit iterated process. If we wish to obtain the dependence of $z_{n+2}$ on $z_{n}$, then from (46) it follows that

$$
\begin{array}{r}
z_{n+2}=\operatorname{Lam}\left[\operatorname{Lam}\left(\exp \left(\lambda-\mu z_{n}\right)\right)\right. \\
\left.\cdot\left(\exp \left(\lambda-\mu \operatorname{Lam}\left(z_{n} \exp \left(\lambda-\mu z_{n}\right)\right)\right)\right)\right], \\
n=0,1,2, \ldots,
\end{array}
$$

and so on.

\section{Other Discrete Maps Describing Behavior of System (31)}

In this section we represent new discrete processes determining the chaotic dynamics of system (31).

It is well known that infinite number of changes of a sign of derivative at least for one of the phase variables at $t \rightarrow \infty$ in any nonlinear system of ordinary differential equations, the appearance of chaos in such system is a necessary condition. Precisely this condition $(\dot{x}(t)=0)$ was fixed as the basis of construction of process (46). Below the cases $\dot{\rho}(t)=0$ and $\dot{\xi}(t)=0$ will also be considered.

(i) Let $\dot{\rho}(t)=0$. Then from the second equation of system (31), it follows that

$$
q \cdot(\cos \xi(t)) x(t)+b \rho(t)+p x(t) \rho(t)=0 .
$$

The period of function $\cos \xi(t)$ is $2 \pi$. Assume that $t_{0}, t_{1}, \ldots, t_{i}, \ldots$ are the sequential points of maximums of the function $\rho(t)$. Let $\rho_{i}=\rho\left(t_{i}\right)$ be the maximums of $\rho(t)$. By $x_{i}=x\left(t_{i}\right)\left(\xi_{i}=\xi\left(t_{i}\right)\right)$ denote the values of the function $x(t)(\xi(t))$ at the points $t_{i} ; i=0,1,2, \ldots$ Then from (47) and the condition $\xi_{i+1}-\xi_{i}=2 \pi$, we have

$$
q \cos \xi_{i}=-\frac{\left(b+p x_{i}\right) \rho_{i}}{x_{i}}
$$


and

$\frac{\left(b+p x_{i}\right) \rho_{i}}{x_{i}}=\frac{\left(b+p x_{i+1}\right) \rho_{i+1}}{x_{i+1}} ; \quad i=0,1, \ldots$

Introduce the designation $\Delta=t_{i+1}-t_{i}>0$. The first equation of system (31) has the solution

$$
\begin{aligned}
& x(t)=x_{0} \exp (a t)+\int_{0}^{t} \exp (a(t-\tau)) \\
& \times(s+d \sin (2 \xi(\tau)+\alpha-2 \beta)) \rho^{2}(\tau) d \tau ; \\
& t>\tau \text {. }
\end{aligned}
$$

Taking into account the introduced designation $\Delta$ from the last relation, we get

$$
\begin{aligned}
x_{i+1}- & \exp (a \Delta) x_{i} \\
= & \int_{0}^{t_{i+1}} \exp \left(a\left(t_{i+1}-\tau\right)\right) \\
& \times(s+d \sin (2 \xi(\tau)+\alpha-2 \beta)) \rho^{2} \\
& \times(\tau) d \tau-\exp (a \Delta) \int_{0}^{t_{i}} \exp \left(a\left(t_{i}-\tau\right)\right) \\
& \times(s+d \sin (2 \xi(\tau)+\alpha-2 \beta)) \rho^{2}(\tau) d \tau .
\end{aligned}
$$

Let $f(t)=(s+d \sin (2 \xi(t)+\alpha-2 \beta)) \rho^{2}(t)$. Suppose that $t=t_{i} \rightarrow \infty$. Consider the chain of transformations of formula (49):

$$
\begin{aligned}
x_{i+1}- & \exp (a \Delta) x_{i} \\
= & \exp (a(t+\Delta)) \int_{0}^{t+\Delta} \exp (-a \tau) f(\tau) d \tau \\
& -\exp (a(t+\Delta)) \int_{0}^{t} \exp (-a \tau) f(\tau) d \tau \\
= & \exp (a(t+\Delta)) \int_{t}^{t+\Delta} \exp (-a \tau) f(\tau) d \tau .
\end{aligned}
$$

We again apply the second theorem about the average value to the last relation. Then we get

$$
\begin{aligned}
x_{i+1}- & \exp (a \Delta) x_{i} \\
= & \exp (a(t+\Delta)) \int_{t_{i}}^{t_{i+1}} \exp (-a \tau) \\
& \times(s+d \sin (2 \xi(\tau)+\alpha-2 \beta)) \rho^{2}(\tau) d \tau \\
= & \rho^{2}\left(t_{i}\right) \exp (a(t+\Delta)) \int_{t_{i}}^{\xi_{i}} \exp (-a \tau) \\
& \times(s+d \sin (2 \xi(\tau)+\alpha-2 \beta)) d \tau
\end{aligned}
$$

$$
\begin{aligned}
& +\rho^{2}\left(t_{i+1}\right) \exp (a(t+\Delta)) \int_{\zeta_{i}}^{t_{i+1}} \exp (-a \tau) \\
& \times(s+d \sin (2 \xi(\tau)+\alpha-2 \beta)) d \tau \\
= & \alpha_{2 i} \rho_{i}^{2}+\alpha_{2 i+1} \rho_{i+1}^{2} ; \quad i=0,1, \ldots
\end{aligned}
$$

Here $t_{i} \leq \xi_{i} \leq t_{i, i+1}, t_{i, i+1} \leq \zeta_{i} \leq t_{i+1}$, and

$$
\begin{aligned}
\alpha_{2 i} & =\exp (a(t+\Delta)) \int_{t_{i}}^{t_{i, i+1}} \exp (-a \tau) \gamma(\tau) d \tau \\
& \leq \frac{s+|d|}{-a}(1-\exp (a \Delta)), \\
\alpha_{2 i+1} & =\exp (a(t+\Delta)) \int_{t_{i, i+1}}^{t_{i+1}} \exp (-a \tau) \gamma(\tau) d \tau \\
& \leq \frac{s+|d|}{-a}(1-\exp (a \Delta)) ; \\
\gamma(\tau) & =(s+d \sin (2 \xi(\tau)+\alpha-2 \beta))>0 .
\end{aligned}
$$

We can consider that $\alpha_{2 i}=\alpha_{0}, \alpha_{2 i+1}=\alpha_{1}$. In this case, the formula (49) can be rewritten in the form

$$
x_{i+1}=\exp (a \Delta) x_{i}+\alpha_{0} \rho_{i}^{2}+\alpha_{1} \rho_{i+1}^{2} .
$$

We also introduce the designations: $\lambda=$ $\exp (a \Delta)$ (since $a<0$, then $\lambda<1$ ), and

$$
u_{i}=\frac{-p}{b} x_{i}, \quad v_{i}=\frac{\sqrt{-p}}{\sqrt{b}} \rho_{i} .
$$

Then combining relations (48) and (50), we get 2D implicit 3-parametric iterated process

$$
\left\{\begin{aligned}
u_{i+1}= & \lambda u_{i}+\alpha_{0} v_{i}^{2}+\alpha_{1} v_{i+1}^{2}, \quad 0<\lambda<1 ; \\
v_{i+1}= & v_{i} \frac{u_{i} \cdot\left(1-u_{i+1}\right)}{u_{i+1} \cdot\left(1-u_{i}\right)}, \\
& 0<u_{i}<1 ; i=0,1, \ldots
\end{aligned}\right.
$$

Here $\lambda, \alpha_{0}$ and $\alpha_{1}$ are positive parameters.

(ii) Let $\dot{\xi}(t)=0$. Then from the third equation of system (31) it follows that

$$
c+q \cdot(\sin \xi(t)) \frac{x(t)}{\rho(t)}=0 .
$$

We will consider that for some $q \in R$, Eq. (52) has a solution $t^{*} \in[0, \infty]$.

Applying reasonings of item (i) to (52), we obtain such relation

$$
\frac{x_{i}}{\rho_{i}}=\frac{x_{i+1}}{\rho_{i+1}} ; \quad i=0,1,2, \ldots
$$


Note that the points of maximums of the function $\xi(t)$ do not coincide with the points of maximums of the function $\rho(t)$ (or $x(t)$ ). However at the conclusion of formula (50) this fact was not used. Therefore, it is possible to use this formula. In total, we get

$$
\left\{\begin{array}{l}
u_{i+1}=\lambda u_{i}+\alpha_{0} v_{i}^{2}+\alpha_{1} v_{i+1}^{2}, \quad 0<\lambda<1 \\
v_{i+1}=v_{i} \frac{u_{i+1}}{u_{i}} ; \quad i=0,1, \ldots
\end{array}\right.
$$

We mark that sequences $u_{i}, v_{i}$ in processes (51) and (53) are different.

It is clear that 2D maps (51) and (53) are more complex than 1D map (46). On the other hand, maps (51) and (53) are rational while (46) is exponential. (Notice that system (53) can be reduced to the explicit form: $u_{i+1}=\varphi_{1}\left(u_{i}, v_{i}\right)$; $v_{i+1}=\varphi_{2}\left(u_{i}, v_{i}\right)$.)

\section{Examples}

Consider the following system

$$
\left\{\begin{array}{l}
\dot{x}(t)=-2 x(t)+7 y^{2}(t)+13 z^{2}(t), \\
\dot{y}(t)=q x(t)+7 y(t)+10 z(t)-3 x(t) y(t), \\
\dot{z}(t)=-10 y(t)+7 z(t)-3 x(t) z(t) .
\end{array}\right.
$$

In the polar coordinates system (54) takes the form [see (8)]

$$
\left\{\begin{array}{l}
\dot{x}(t)=-2 x+(10-3 \cdot \cos 2 \xi) \rho^{2}, \\
\dot{\rho}(t)=q \cdot(\cos \xi) \cdot x+7 \rho-3 x \rho, \\
\dot{\xi}(t)=-10-q \cdot(\sin \xi) \cdot \frac{x}{\rho}
\end{array}\right.
$$

Let us construct the iterated process (36) for system (54). We have $b=7, c=10$, and $p=3$.

Note that $\forall q \geq 0$ we have

$$
\begin{aligned}
\Psi & =\lim _{k \rightarrow \infty} \frac{\lambda(q)}{\alpha_{0}(q) x_{k}+\alpha_{1}(q) x_{k+1}} \\
& =-\frac{p}{b\left(t_{k+1}-t_{k}\right)} \int_{t_{k}}^{t_{k+1}} x(t) d t \approx 1 .
\end{aligned}
$$

This equality can serve as a criterion for the verification of correctness of calculations.

(1) Let $q=0$. Then for formulas (36) and (44), we obtain: $t_{k}=45.155, t_{k, k+1}=45.312, t_{k+1}=45.469$, and $x_{\max }=x_{k}=x_{k+1}=2.408$. Besides, for system $(55), \xi\left(t_{k}\right)=451.55, \xi\left(t_{k+1}\right)=454.69$ and

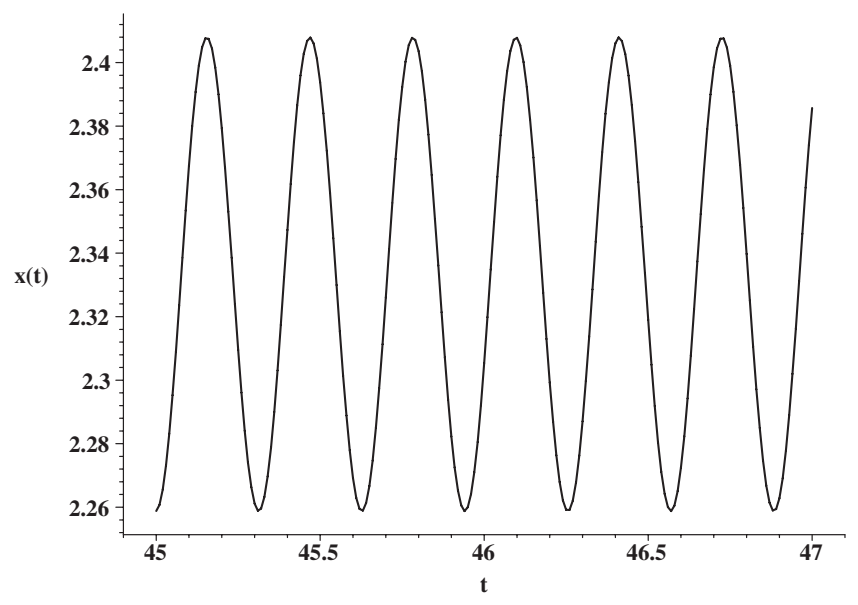

(a)

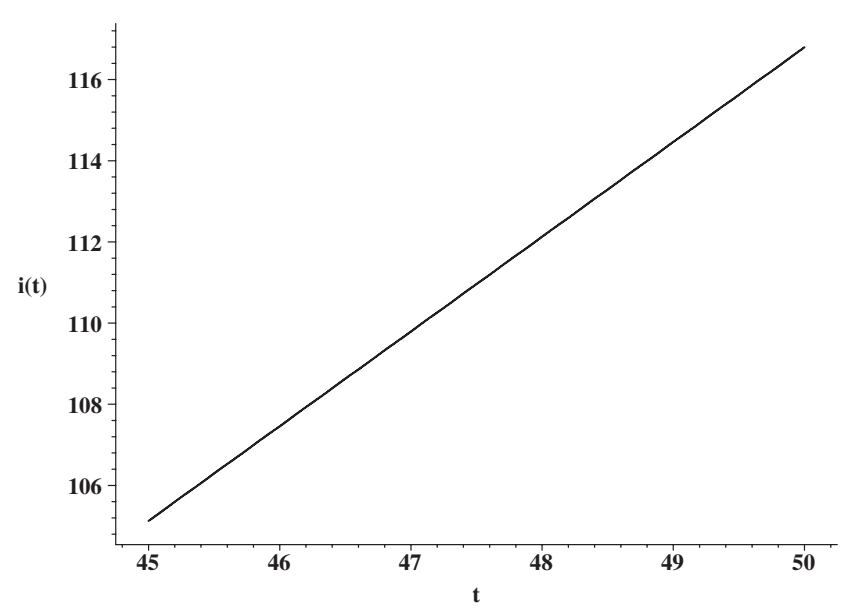

(b)

Fig. 1. The graphs of dependence (a) $x(t)$ and (b) $\int x(t)$ on $t$ for system (54) at $q=0$.

$\xi\left(t_{k+1}\right)-\xi\left(t_{k}\right)=\pi$. From Figs. 1(a) and 1(b), we have:

$$
\begin{gathered}
\alpha_{0}=\alpha_{1}=0.5 \cdot 6 \cdot \frac{i(45.469)-i(45.155)}{x_{k}} \\
=0.5 \cdot 6 \frac{106.222-105.489}{2.408}=0.913 ; \\
x_{k+1}=x_{k} \exp \left(4.396-0.913 x_{k}-0.913 x_{k+1}\right) ; \\
k=0,1, \ldots
\end{gathered}
$$

and $\Psi=0.999$. This is a $\pi$-periodic process. (Here $\varepsilon(q)=0$.)

Now assume that $q \geq 1.65$.

Note that at increase of parameter $q$, the parameter $\lambda$ changes approximately in accord to the formula $\lambda=2 \pi b m / c ; m=1,2,4, \ldots$ However at the values $q \in(1.25-1.26), q \in(1.32-1.33)$, 


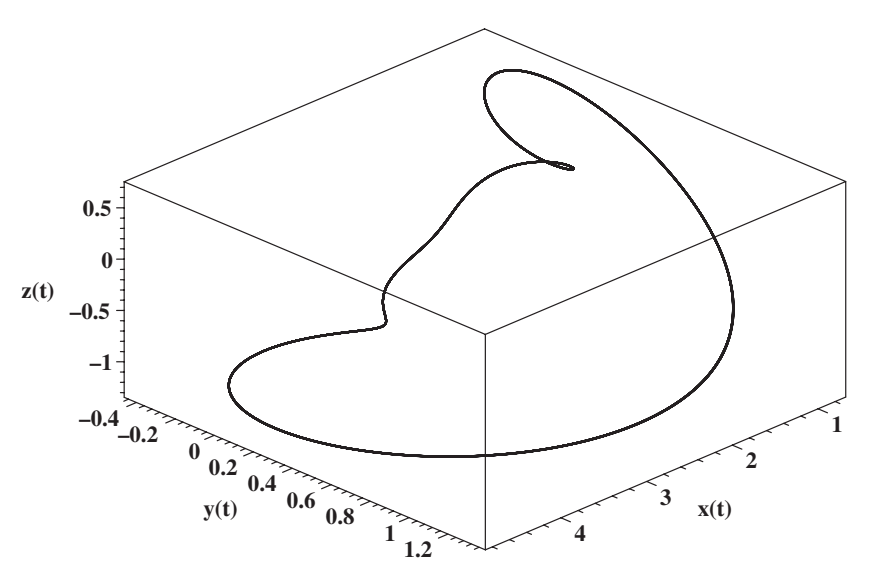

(a)

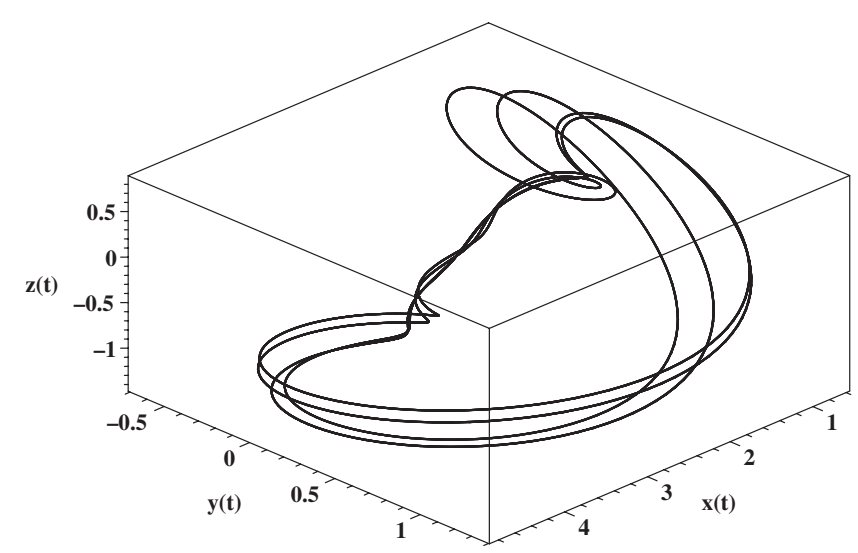

(c)

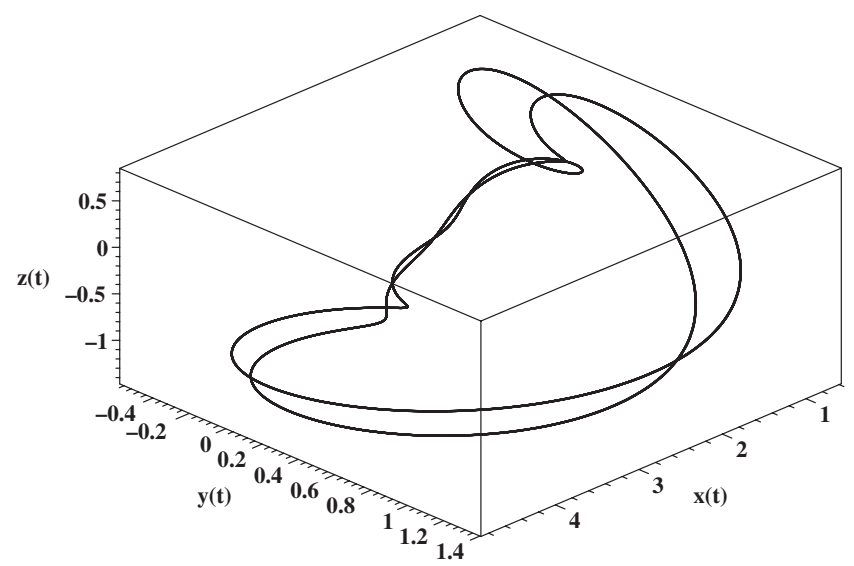

(b)

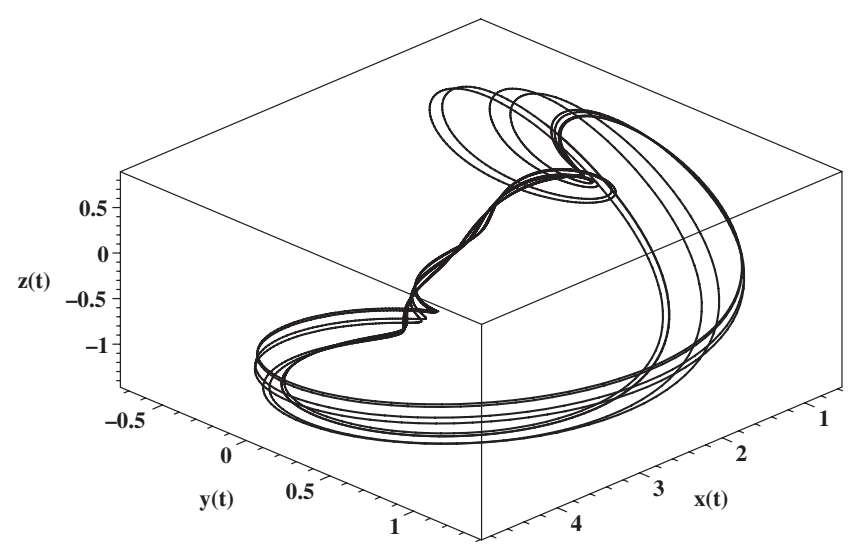

(d)

Fig. 2. The phase portraits of system (54) at different values of $q$. (a) $q=1.65$. There is 1 -cycle, (b) $q=1.7$. There is 2 -cycle, (c) $q=1.71$. There is 4 -cycle and (d) $q=1.713$. There is 8 -cycle.

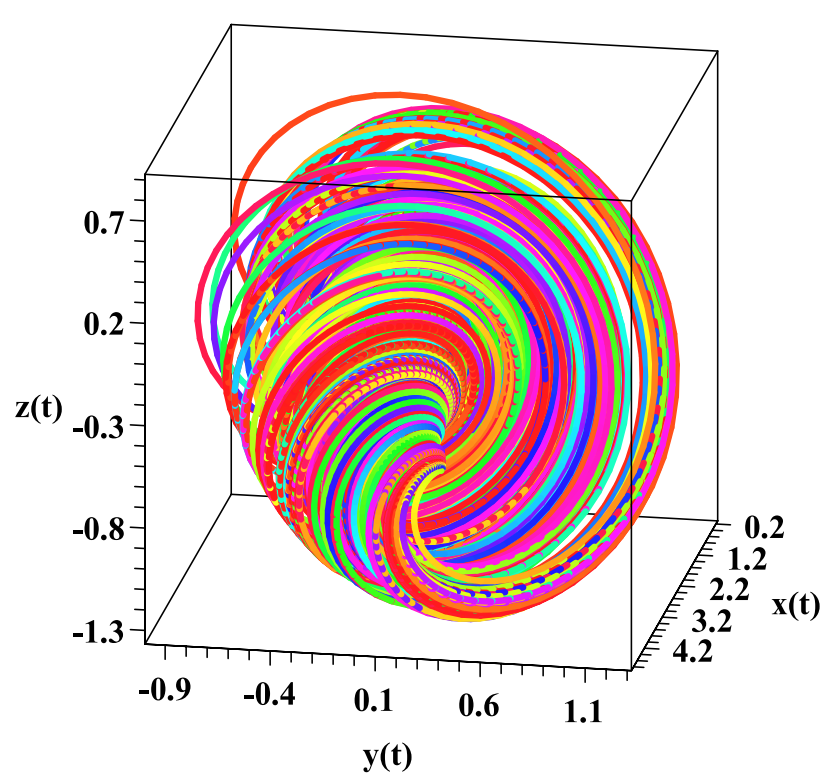

Fig. 3. The phase portrait of system (54) at $q=1.81$. There is chaos. $q \in(1.37-1.38), q \in(1.53-1.54)$, bifurcations take place violating this process.

(2) Let $q=1.65$. From Fig. 4(a) it follows that $t_{k}=30.94, t_{k+2}=33.31, t_{k, k+2}=32.78$, and $x_{\max }=x_{k}=x_{k+2}=4.88$. Besides, for system (55) $\xi\left(t_{k}\right)=-83.157, \xi\left(t_{k+2}\right)=-89.440, \xi\left(t_{k, k+2}\right)=$ -84.693 , and $\xi\left(t_{k+2}\right)-\xi\left(t_{k}\right)=2 \pi$. From Fig. 4(b), we have:

$$
\begin{aligned}
\alpha_{0} & =6 \frac{i(32.78)-i(30.94)}{x_{k}} \\
& =6 \frac{73.975-69.721}{4.88}=5.234 ; \\
\beta_{2} & =6 \frac{i(33.31)-i(32.78)}{x_{k+2}} \\
& =6 \frac{75.225-73.975}{4.88}=1.534 .
\end{aligned}
$$




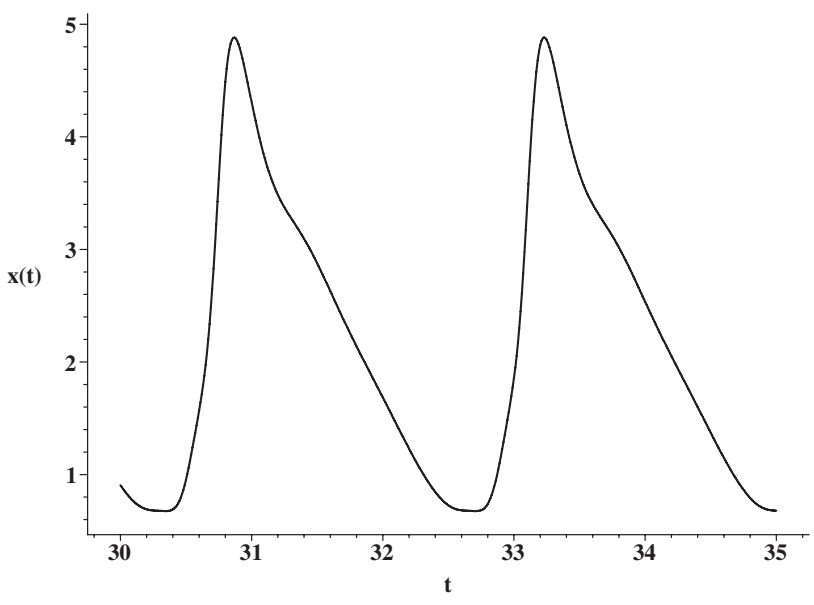

(a)

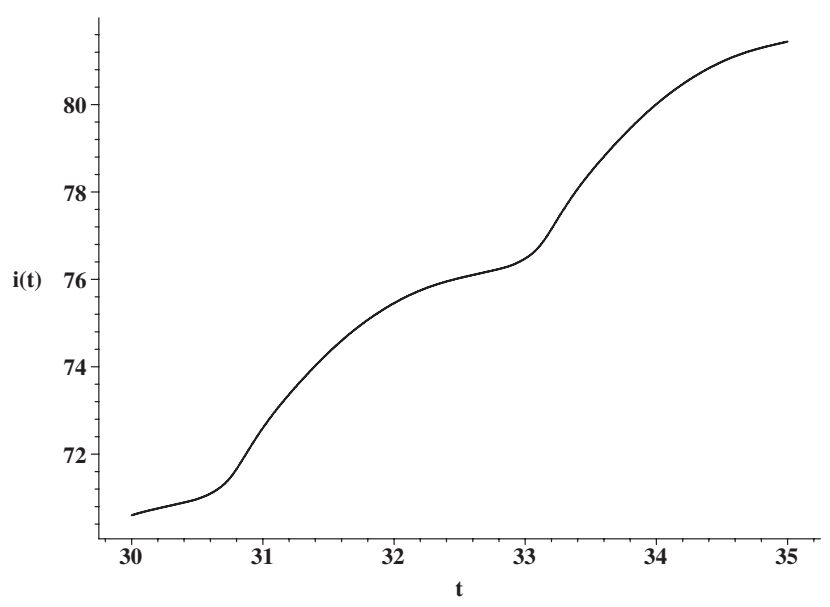

(b)

Fig. 4. The graphs of dependence (a) $x(t)$ and (b) $\int x(t)$ on $t$ for system (54) at $q=1.65$.

Thus, process (45) for system (54) at $q=1.65$ has the form

$$
\begin{array}{r}
x_{k+2}=x_{k} \exp \left(33.180-5.234 x_{k}-1.534 x_{k+2}\right), \\
k=0,1, \ldots .
\end{array}
$$

and $\Psi=1.024$. This is a $2 \pi$-periodic process.

(3) Let $q=1.70$. In this case, a period-doubling bifurcation takes place. Therefore, we take advantage of formulas (44) and (45).

From Fig. 5(a) it follows that $t_{k}=33.62$, $t_{k, k+1}=35.60, t_{k+1}=36.06, t_{k+1, k+2}=37.70$, $t_{k+2}=38.406$, and $x_{\max }=x_{k}=x_{k+2}=4.89$, $x_{\max 1}=x_{k+1}=4.616$. Besides, for system (55)

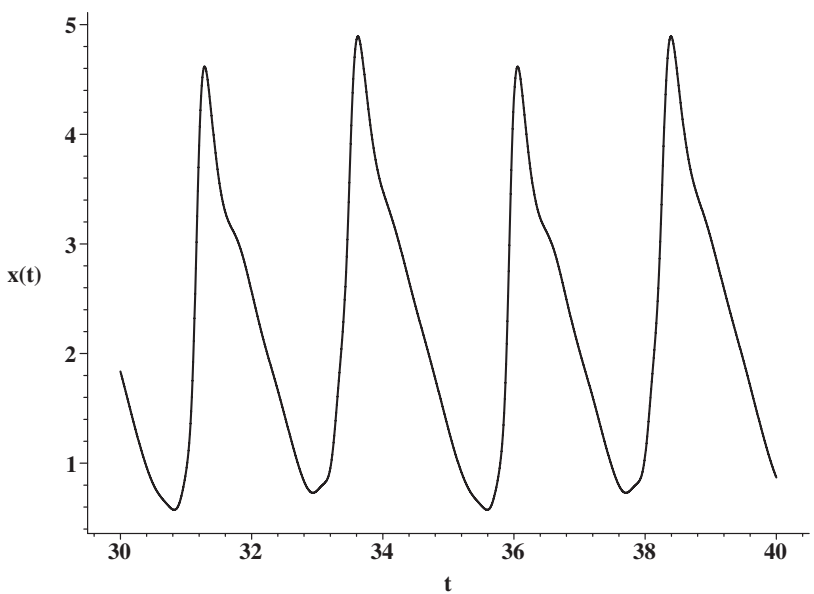

(a) $\xi\left(t_{k}\right)=89.366, \xi\left(t_{k+2}\right)=101.936$ and $\xi\left(t_{k+2}\right)-$ $\xi\left(t_{k}\right)=4 \pi$. From Fig. 4(b), we have:

$$
\begin{aligned}
\alpha_{0} & =6 \frac{i(35.60)-i(33.62)}{x_{k}} \\
& =6 \frac{79.474-74.966}{4.89}
\end{aligned}
$$$$
=5.531 \text {; }
$$

$$
\alpha_{1}=6 \frac{i(36.06)-i(35.60)}{x_{k+1}}
$$

$$
=6 \frac{80.387-79.474}{4.616}
$$

$=1.187$

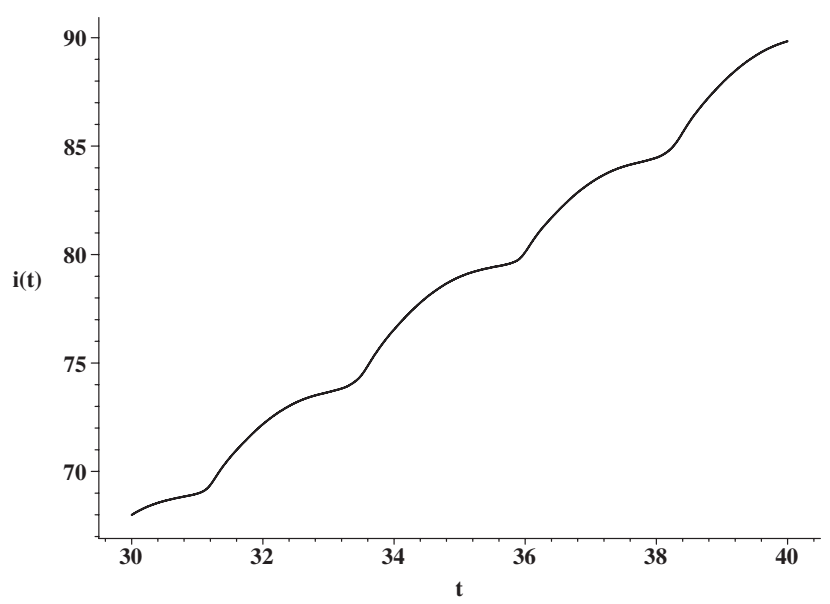

(b)

Fig. 5. The graphs of dependence (a) $x(t)$ and (b) $\int x(t)$ on $t$ for system (54) at $q=1.7$. 


$$
\begin{aligned}
\beta_{1} & =6 \frac{i(37.70)-i(36.06)}{x_{k+1}} \\
& =6 \frac{84.221-80.387}{4.616}=4.886 ; \\
\beta_{2} & =6 \frac{i(38.40)-i(37.70)}{x_{k}} \\
& =6 \frac{85.625-84.221}{4.89}=1.722 .
\end{aligned}
$$

For results calculation, we construct two iterated processes:

$$
\begin{aligned}
x_{k+1}= & x_{k} \cdot \exp \left(34.160-5.531 x_{k}-1.187 x_{k+1}\right), \\
x_{k+2}= & x_{k+1} \cdot \exp \left(32.844-4.886 x_{k+1}\right. \\
& \left.-1.722 x_{k+2}\right) ; \quad k=0,1,2, \ldots
\end{aligned}
$$

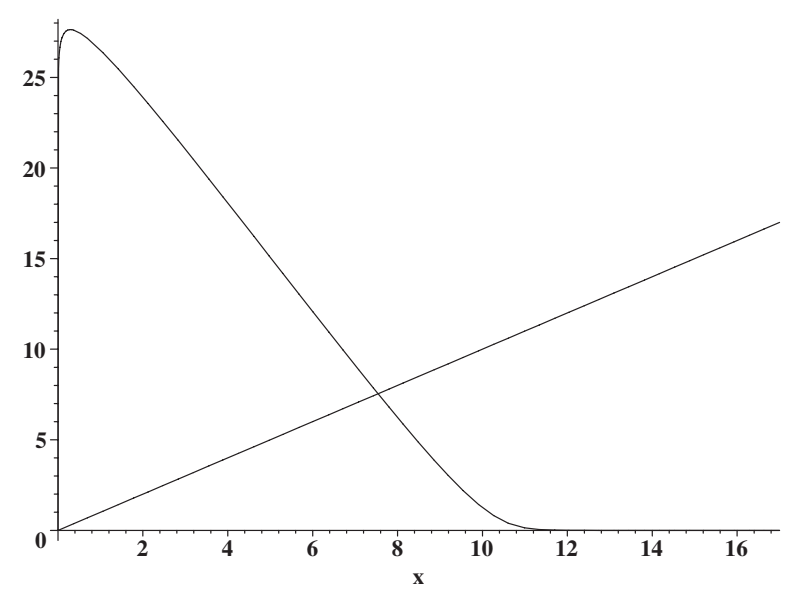

(a)

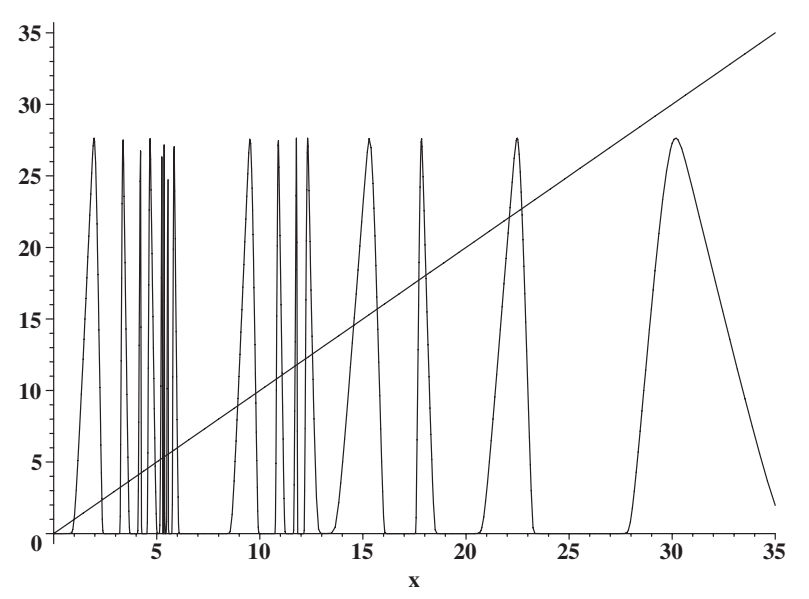

(c)
Thus, process (45) for system (54) at $q=1.70$ has the form

$$
\begin{aligned}
x_{k+2}= & x_{k} \cdot \exp \left(67.004-5.531 x_{k}-1.722 x_{k+2}\right. \\
& \left.-6.06 \cdot \Theta\left(x_{k}, x_{k+2}\right)\right) ; \quad k=0,1,2, \ldots,
\end{aligned}
$$

where $\Theta\left(x_{k}, x_{k+2}\right)$ may be calculated like (45). Here $\Psi=0.96$ and the process is $4 \pi$-periodic.

(4) Let $q=1.81$. In this case, we have: $t_{k}=33.45$, $t_{k+1}=36.20, t_{k, k+1}=35.77 ; x_{k}=4.947, x_{k+1}=$ $3.958 ; i_{k}=69.530, i_{k, k+1}=74.717, i_{k+1}=75.390$.

For results calculation, we construct the iterated process:

$$
\begin{aligned}
x_{k+1}= & x_{k} \cdot \exp \left(38.501-6.291 x_{k}\right. \\
& \left.-1.020 x_{k+1}\right) ; \quad k=0,1, \ldots
\end{aligned}
$$

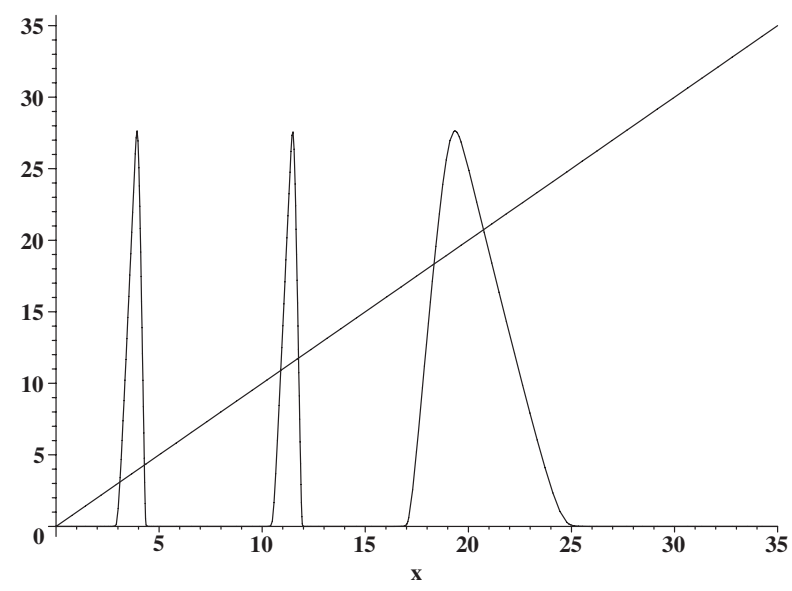

(b)

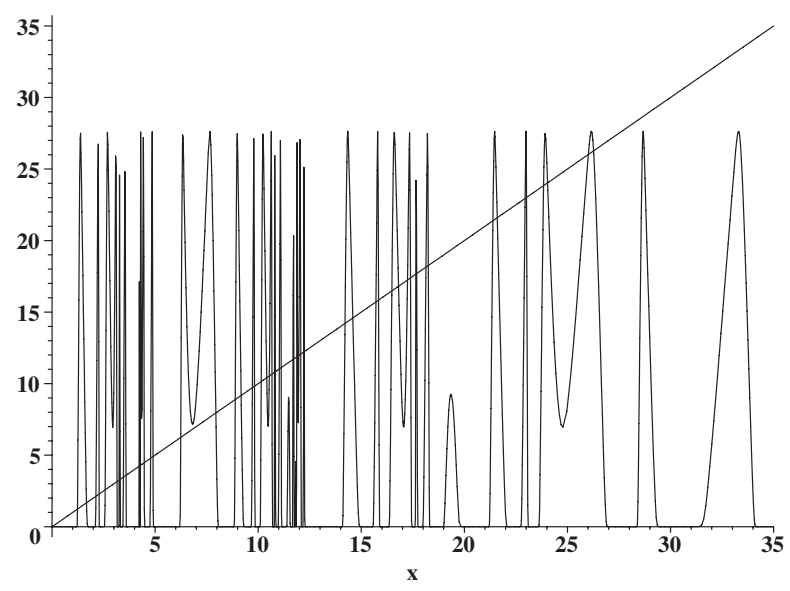

(d)

Fig. 6. The iterations of map (58) and its fixed points at different $n$. (a) $n=1 ; y=f(x)$, (b) $n=4 ; y=f(f(\ldots f(x)))=$ $f^{(4)}(x),\left(\right.$ c) $n=6 ; y=f(f(\ldots f(x)))=f^{(6)}(x)$ and $(\mathrm{d}) n=8 ; y=f(f(\ldots f(x)))=f^{(8)}(x)$. 
Let us compare processes (56) and (57) between themselves. For this purpose, we will take advantage of formula (46). Then we get for processes (56) and (57)

$$
\begin{array}{r}
z_{n+1}=f\left(z_{n}\right) \equiv \operatorname{Lam}\left(z_{n} \exp \left(33.18-3.40 z_{n}\right)\right), \\
n=0,1,2, \ldots, \\
z_{n+1}=g\left(z_{n}\right) \equiv \operatorname{Lam}\left(z_{n} \exp \left(38.50-6.17 z_{n}\right)\right), \\
n=0,1,2, \ldots .
\end{array}
$$

From Fig. 6, it follows that map (58) is not chaotic because at different $n$ a number of fixed points of map $f^{(n)}(x)$ remains limited. At the same time from Fig. 7, it does not follow that map (59)

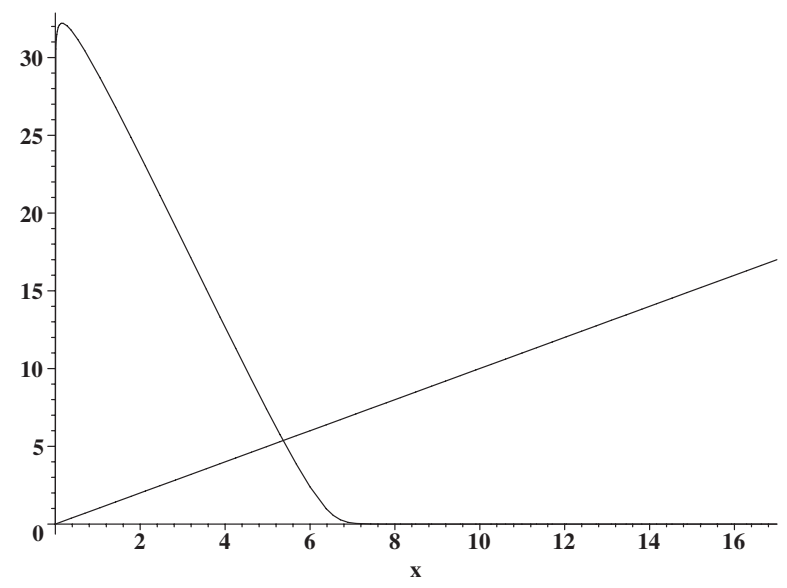

(a)

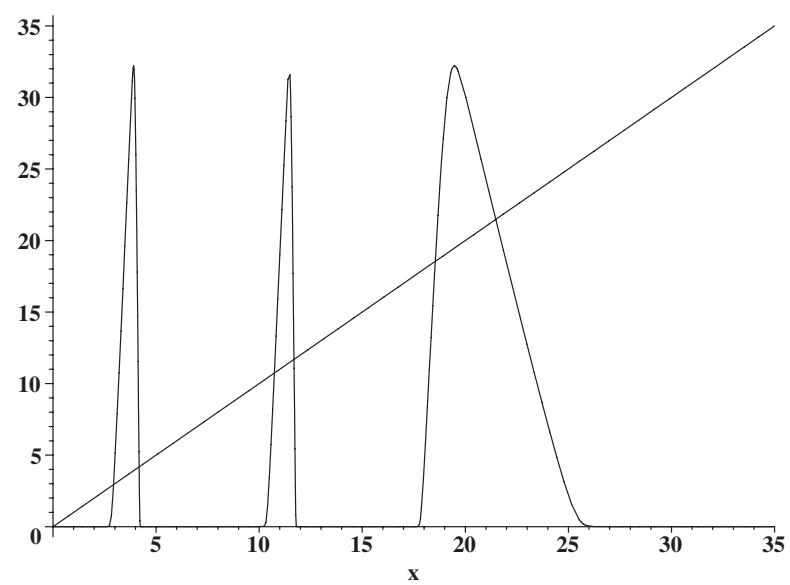

(c) is chaotic. Nevertheless a verification showed that for $n \leq 10$ the number of fixed points of the map $g^{(n)}(x)$ is multiplied with the growth $n$.

Let us build a bifurcation diagram of map (46) at $\mu=6.17$ and $\lambda \in(30,40)$.

From Fig. 8 it is clear that the map $g(z)$ is chaotic at $\lambda=38.5$. It means chaotic of system (55) [or (54)] at $q=1.81$.

Finally, we note that Theorem 6 for all indicated values of parameters $a=-2, a_{12}=a_{13}=0$, $b_{11}=7, b_{22}=13, c_{12}=c_{21}=0, c_{11}=c_{22}=-3$, $a_{22}=7$, and $a_{23}=10$ of system (54) remains valid at $0<q \leq 2.27$. (At the indicated values of $q$ the point $(0,0,0)$ is a unique equilibrium point of system (54).)

The analysis of the resulting examples shows that a dependence of parameters $\lambda, \alpha_{0}$, and $\alpha_{1}$ on

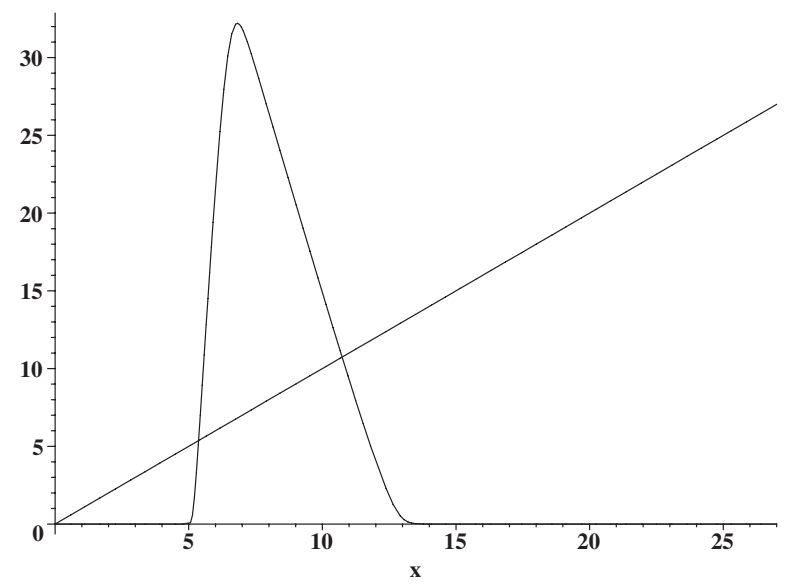

(b)

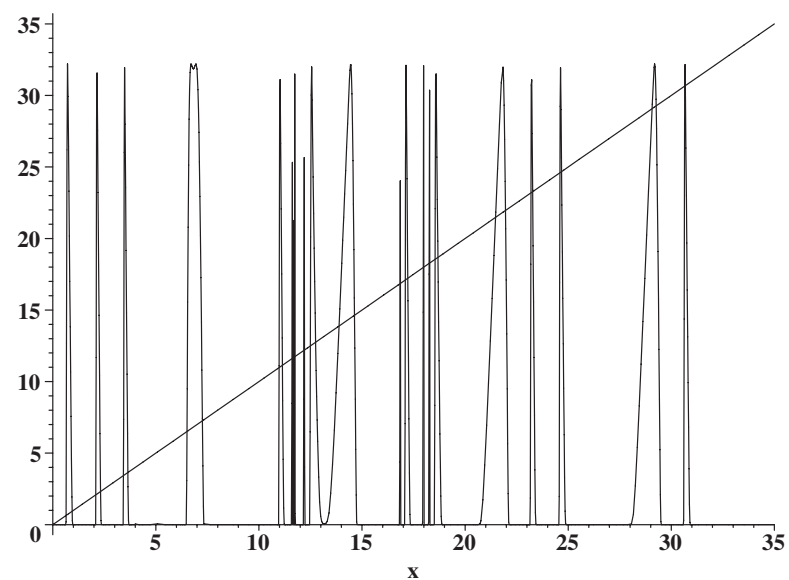

(d)

Fig. 7. The iterations of map (59) and its fixed points at different $n$. (a) $n=1 ; y=g(x),(\mathrm{b}) n=2 ; y=g(g(x))=g^{(2)}(x)$, (c) $n=4 ; y=g(g(\ldots g(x)))=g^{(4)}(x)$ and $(\mathrm{d}) n=8 ; y=g(g(\ldots g(x)))=g^{(8)}(x)$. 


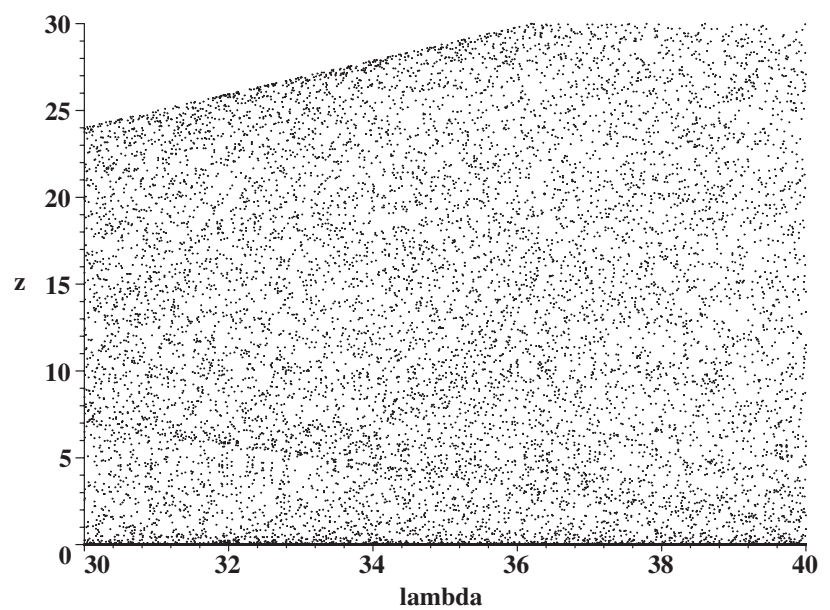

Fig. 8. The bifurcation diagram of the map $g(z)=$ $\operatorname{Lam}(z \exp (\lambda-6.17 z))$.

$q$ in process (36) at other fixed parameters of system (54) [or (31)] is continuously discrete. For the continuous change of parameter $q$ on the interval (1.54-1.69), period $T_{1}$ is $2 \pi$; if $q \in(1.69-1.71)$ then period $T_{2}=4 \pi$; if $q \in(1.71-1.713)$ then period $T_{3}=8 \pi$. Chaos is observed at $q \in(1.74-1.83)$. Besides, we note that in all considered examples the function $\varepsilon(q)=0$ or $\varepsilon(q) \approx 0$.

\section{Conclusion}

- There are a few methods to prove the existence of chaotic attractors in nonlinear dynamic systems. For example, in [Tucker, 1999] the proof of existence of the Lorenz attractor is based on a combination of normal form theory and rigorous numerical computations. In the present work, another idea was developed: for the quadratic 3D system a suitable discrete 1D mapping [(36) or (46)] is built; its chaoticity is then proved. In our opinion there is a more simple way of proof of existence of chaotic dynamics in system (6).

- The method allows the known quadratic 3D system (6) to construct the quadratic 2D system (4) such that from boundedness of the solutions of system (4) follows the boundedness of the solutions of system (6) being offered.

- It is shown that chaotic dynamics in system (4) at $a_{21}=a_{31}=0$ and in that system at $a_{21} \neq 0$ or $a_{31} \neq 01$ is described by the same discrete map (36). In addition, it is shown as with the help of the Lambert function to pass from the implicit process (36) to the explicit process (46).
- The present work, in which was assumed that the discriminant Disc $<0$, is connected to works [Belozyorov, 2011b, 2012], where Disc > 0 was assumed and establishes the theoretical bases for research on chaotic process in system (6).

- The study of discrete map (46) depending on the parameters $\lambda$ and $\mu$ is of independent interest. In the future the author hopes to get back to researching the properties of its map.

\section{Acknowledgments}

The author expresses his sincere gratitude to the anonymous reviewers calling his attention to the incompleteness of the proof of Theorem 4. In the last variant of the manuscript, this defect was removed.

\section{References}

Bao, J. \& Yang, Q. [2011] "A new method to find homoclinic and heteroclinic orbits," Appl. Math. Comput. 217, 6526-6540.

Belozyorov, V. Ye. [2011a] "On existence of homoclinic orbits for some types of autonomous quadratic systems of differential equations," Appl. Math. Comput. 217, 4582-4595.

Belozyorov, V. Ye. [2011b] "New types of 3-D systems of quadratic differential equations with chaotic dynamics based on Ricker discrete population model," Appl. Math. Comput. 218, 4546-4566.

Belozyorov, V. Ye. [2012] "Implicit one-dimensional discrete maps and their connection with existence problem of chaotic dynamics in 3-D systems of differential equations," Appl. Math. Comput. 218, 8869-8886.

Belozyorov, V. Ye. \& Chernyshenko, S. V. [2013] "Generating chaos in 3D systems of quadratic differential equations with 1D exponential maps," Int. J. Bifurcation and Chaos 23, 1350105.

Chen, B., Zhou, T. \& Chen, G. [2009] "An extended Shilnikov homoclinic theorem and its applications," Int. J. Bifurcation and Chaos 19, 1679-1693.

Dickson, R. J. \& Perko, L. M. [1970] "Bounded quadratic systems in the plane," J. Diff. Eqs. 7, 251-273.

El-Dessoky, M. M., Yassen, M. T., Saleh, E. \& Aly, E. S. [2012] "Existence of heteroclinic and homoclinic orbits in two different chaotic dynamical systems," Appl. Math. Comput. 218, 11859-11870.

Feng, J. \& Tse, C. K. [2007] Reconstruction of Chaotic Signals with Applications to Chaos-Based Communications (World Scientific, Singapore, Tsinghua University Press, Beijing). 
Gardini, L., Sushko, I., Avrutin, V. \& Schanz, M. [2011] "Critical homoclinic orbits lead to snap-back repellers," Chaos Solit. Fract. 44, 433-449.

Gunay, E. \& Kilic, R. [2011] "A new way of generating $N$-scroll attractors via trigonometric function," Int. J. Bifurcation and Chaos 21, 897-901.

Jiang, Y. \& Sun, J. [2007] "Shilnikov homoclinic orbits in a new chaotic system," Chaos Solit. Fract. 32, $150-159$.

Khalil, H. K. [1996] Nonlinear Systems, 2nd edition (Prentice Hall, NJ).

Khan, A. \& Kumar, P. [2013] "Chaotic properties on time varying map and its set valued extension," Adv. Pure Math. 3, 359-364.

Kocan, Z. [2012] "Chaos on one-dimensional compact metric spaces," Int. J. Bifurcation and Chaos 22, 1250259.

Kuznetsov, Y. A. [1998] Elements of Applied Bifurcation Theory, 2nd edition (Springer-Verlag, NY).

Leonov, G. A. [2012] "General existence conditions of homoclinic trajectories in dissipative systems. Lorenz, Shimizu-Morioka, Lu and Chen systems," Phys. Lett. A 376, 3045-3050.

Leonov, G. A. [2013] "Shilnikov chaos in Lorenz-like systems," Int. J. Bifurcation and Chaos 23, 1350058.

Leonov, G. A. \& Kuznetsov, N. V. [2013] "Hidden attractors in dynamical systems. From hidden oscillations in Hilbert-Kolmogorov, Aizerman, and Kalman problems to hidden chaotic attractor in Chua circuits," Int. J. Bifurcation and Chaos 23, 1330002.

Luo, A. C. J. \& Guo, Y. [2010] "Parameter characteristics for stable and unstable solutions in nonlinear discrete dynamical systems," J. Diff. Eqs. 20, 3173-3191.

Peng, C. C. [2008] "Rigorous verification of the existence of transversal homoclinic orbits," Int. J. Bifurcation and Chaos 18, 793-801.

Robinson, R. C. [2004] An Introduction to Dynamical Systems: Continuous and Discrete (Prentice Hall/NY, London).

Shang, D. \& Han, M. [2005] "The existence of homoclinic orbits to saddle-focus," Appl. Math. Comput. 163, 621-631.

Shen, X. \& Jia, Z. [2011] "On the existence structure of one-dimensional discrete chaotic systems," J. Math. Res. 3, 22-27.

Tucker, W. [1999] "The Lorenz attractor exists," C. R. Acad. Sci. Paris 328, Ser. I, 1197-1202.

Wang, X. [2009] "Shilnikov chaos and Hopf bifurcation analysis of Rucklidge system," Chaos Solit. Fract. 42, 2208-2217.

Yang, Q., Wei, Z. \& Chen, G. [2010] "An unusual 3D autonomous quadratic chaotic system with two stable node-foci," Int. J. Bifurcation and Chaos 20, 10611083.

Zhang, X., Shi, Y. \& Chen, G. [2009] "Constructing chaotic polynomial maps," Int. J. Bifurcation and Chaos 19, 531-543.

Zheng, Z. \& Chen, G. [2006] "Existence of heteroclinic orbits of the Shilnikov type in a 3 -D quadratic autonomous chaotic systems," J. Math. Anal. Appl. 315, 106-119. 